\title{
Protective Effects of Flavonoids in Acute Models of Light-Induced Retinal Degeneration ${ }^{\circledR}$
}

\author{
Joseph T. Ortega, Tanu Parmar, Marcin Golczak, and (B) Beata Jastrzebska \\ Department of Pharmacology, Cleveland Center for Membrane and Structural Biology, School of Medicine, Case Western \\ Reserve University, Cleveland, Ohio
}

Received May 19, 2020; accepted October 26, 2020

\begin{abstract}
Degeneration of photoreceptors caused by excessive illumination, inherited mutations, or aging is the principal pathology of blinding diseases. Pharmacological compounds that stabilize the visual receptor rhodopsin and modulate the cellular pathways triggering death of photoreceptors could avert this pathology. Interestingly, flavonoids can modulate the cellular processes, such as oxidative stress, inflammatory responses, and apoptosis, that are activated during retinal degeneration. As we found previously, flavonoids also bind directly to unliganded rod opsin, enhancing its folding, stability, and regeneration. In addition, flavonoids stimulate rhodopsin gene expression. Thus, we evaluated the effect of two main dietary flavonoids, quercetin and myricetin, in ATP-binding cassette subfamily $\mathrm{A}$ member $4^{-1-} /$ retinol dehydrogenase $8^{-9-}$ and wild-type BALB/c mice susceptible to light-induced photoreceptor degeneration. Using in vivo imaging, such as optical coherence tomography, scanning laser ophthalmoscopy, and histologic assessment of retinal morphology, we found that treatment with these flavonoids prior to light insult remarkably protected retina from deterioration and preserved its function. Using highperformance liquid chromatography-mass spectrometry analysis, we detected these flavonoids in the eye upon their intraperitoneal
\end{abstract}

\section{Introduction}

The visual receptors, rhodopsin and cone opsins expressed in rod and cone photoreceptors in the retina, absorb light photons, which then are converted into a neural impulse. Both

This research was supported in part by grants from National Institutes of Health (NIH) National Eye Institute [Grant R01-EY025214] (to B.J.), [Grant R01-EY023948] (to M.G.).

Conflicts of Interest

The authors declare that they have no conflicts of interest with the contents of this article.

https://doi.org/10.1124/molpharm.120.000072.

S This article has supplemental material available at molpharm. aspetjournals.org. administration. The molecular events associated with the protective effect of quercetin and myricetin were related to the elevated expression of photoreceptor-specific proteins, rhodopsin and cone opsins, decreased expression of the specific inflammatory markers, and the shift of the equilibrium between cell death regulators $B C L 2$-associated $X$ protein (BAX) and B-cell lymphoma 2 toward an antiapoptotic profile. These results were confirmed in photoreceptor-derived $661 \mathrm{~W}$ cells treated with either $\mathrm{H}_{2} \mathrm{O}_{2}$ or all-trans-retinal stressors implicated in the mechanism of retinal degeneration. Altogether, flavonoids could have significant prophylactic value for retinal degenerative diseases.

\section{SIGNIFICANCE STATEMENT}

Flavonoids commonly present in food exhibit advantageous effects in blinding diseases. They bind to and stabilize unliganded rod opsin, which in excess accelerates degenerative processes in the retina. Additionally, flavonoids enhance the expression of the visual receptors, rod and cone opsins; inhibit the inflammatory reactions; and induce the expression of antiapoptotic markers in the retina, preventing the degeneration in vivo. Thus, flavonoids could have a prophylactic value for retinal degenerative diseases.

ABBREVIATIONS: ABCA4, ATP-binding cassette subfamily A member 4; A2E, bis-retinoid $N$-retinyl- $N$-retinylidene ethanolamine; AF, autofluorescence; AMD, age-related macular degeneration; BAX, BCL2-associated X protein; BCL-2, B-cell lymphoma 2; CCL2, chemokine 2; DAPI, 4'6'-diamidino-2-phenyl-indole; DHE, dihydroethidium; ERG, electroretinography; GAPDH, glyceraldehyde-3-phosphate dehydrogenase; GFAP, glial fibrillary acidic protein; HPLC, high-performance liquid chromatography; IL6, interleukin 6; m/z, mass-to-charge ratio; Mef2c, protein myocyte enhancer factor 2c; MS, mass spectrometry; MTT, 3-(4,5-dimethyl-2-thiazolyl)-2,5-diphenyl-2H-tetrazolium bromide; OCT, optical coherence tomography; ONH, optic nerve head; ONL, outer nuclear layer; PCR, polymerase chain reaction; PNA, peanut agglutinin; PVDF, polyvinylidene difluoride; RDH8, retinol dehydrogenase 8; ROS, reactive oxygen species; RPE, retinal pigment epithelium; RPE65, retinal pigment epithelium-specific 65-kDa protein; RT, room temperature; RT-qPCR, real-time quantitative PCR; SD-OCT, spectral domain-OCT; SLO, scanning laser ophthalmoscopy; TNF $\alpha$, tumor necrosis factor $\alpha$; TUNEL, terminal deoxynucleotidyl transferase 2'-deoxyuridine 5'-triphosphate nick-end labeling; WT, wild type. 
all-trans-retinal accumulates and forms toxic metabolites detrimental to the health of the retina (Fishkin et al., 2005; Kim et al., 2007; Maeda et al., 2008; Sparrow et al., 2010; Chen et al., 2012).

The molecular pathology of the photoreceptors' death caused by excessive illumination is associated with changes in their cellular metabolism and overproduction of reactive oxygen species (ROS) that cause damage to DNA, proteins, and lipids present in the retinal tissue (Chen et al., 2012; Gao et al., 2018; Kaarniranta et al., 2019). Consequently, cellular apoptotic pathways are activated, triggering the elimination of photoreceptors (Sawada et al., 2014). In addition, lightinduced damage leads to enhanced expression of proinflammatory chemokines, which stimulates migration of microglia and macrophages to clear dying photoreceptors (Kohno et al., 2013, 2014; Rashid et al., 2018). Such imbalanced homeostasis in the retina associated with oxidative stress and exacerbated inflammatory responses is the major mechanism contributing to several retinal degenerative disorders, including agerelated macular degeneration (AMD), Stargardt disease, and retinitis pigmentosa (Rashid et al., 2019).

Currently, therapeutic interventions for the retinal degenerative diseases are limited, stressing the necessity for developing new medications to provide more effective and safe therapies. Recent studies indicate that polyphenolic compounds, especially flavonoids, could be viable drug candidates since they may have a role in visual signal transduction and the regeneration of the visual pigment. Moreover, flavonoids possess antioxidant, anti-inflammatory, and antiapoptotic capacity. Indeed, flavonoid-rich fruits and vegetables have beneficial effects in improving sight in several eye-related diseases (Kalt et al., 2010; Huynh et al., 2013; Saw et al., 2014).

As we recently reported, flavonoids, such as quercetin and myricetin, enhance rod opsin stability, increase the rate of retinal binding to ligand-free opsin, enhance the expression of rhodopsin, and improve its membrane integration in vitro (Ortega et al., 2019). Rhodopsin is a critical protein for retinal structural integrity. Thus, an increase in the expression of rhodopsin could be one possible mechanism contributing to the protective effects of flavonoids preventing the degeneration of photoreceptors and deterioration of the retina. The expression of photoreceptor-specific proteins is highly regulated. One of the main transcriptional factors that regulate gene expression in photoreceptors under normal and pathologic conditions is the protein myocyte enhancer factor $2 \mathrm{c}$ (Mef2c) (Hao et al., 2011). Interestingly, flavonoids can modulate the expression of Mef2c in endothelial cells (Patel et al., 2019). However, it is not entirely clear whether they have the same effect on Mef2c expression in the eye.

Despite quite extensive studies on the beneficial effects of flavonoids, the mechanism of their protective effects against light-induced retinal damage is not fully understood. Thus, in this study, we evaluated the effect of quercetin and myricetin in ATP-binding cassette subfamily A member 4 (Abca4) ${ }^{-1-}$ (retinol dehydrogenase 8) $R d h 8^{-I^{-}}$mice, a model of Stargardt disease, a juvenile form of AMD (Maeda et al., 2008, 2014), and wild-type (WT) BALB/c mice (LaVail et al., 1987a,b), which are both susceptible to bright light-induced photoreceptor degeneration through activation of oxidative stress, inflammatory responses, and apoptosis. Several studies have shown that $A b c a 4^{-I-} R d h 8^{-I-}$ mice develop retinal degeneration after illumination with bright light because of both rod and cone photoreceptors' death (Maeda et al., 2008; Chen et al., 2012; Gao et al., 2018). These mice lack two key enzymes, ABCA4 transporter and RDH8, that are involved in all-transretinal metabolism. Exposure to intense light leads to a release of all-trans-retinal from rhodopsin and cone opsin receptors, resulting in an increase of its concentration in the photoreceptor cells. When clearance of released all-trans-retinal is impaired, toxic byproducts, such as all-trans-retinal dimer and bis-retinoid $N$-retinyl- $N$-retinylidene ethanolamine (A2E), accumulate, triggering imbalance in ROS production and activation of an inflammatory response (Chen et al., 2012; Zhao et al., 2017). Thus, these mice exhibit many hallmarks of human juvenile macular degeneration. To unravel the molecular details of quercetin and myricetin actions, we also tested their effects on photoreceptor-derived $661 \mathrm{~W}$ cells exposed to alltrans-retinal and $\mathrm{H}_{2} \mathrm{O}_{2}$-induced oxidative stress (Sharma and Rohrer, 2007; Kanan et al., 2008).

\section{Materials and Methods}

Chemicals and Reagents. Alexa Fluor 555-conjugated goat anti-mouse and Alexa Fluor 555-conjugated goat anti-rabbit secondary antibodies for the immunostaining and $44^{\prime} 6^{\prime}$-diamidino-2-phenylindole (DAPI) for the nuclear staining were purchased from Life Technologies (Grand Island, NY). DMSO was obtained from Sigma (St. Louis, MO). EDTA-free protease inhibitor cocktail tablets were purchased from Roche (Basel, Switzerland). Myricetin, quercetin, and 3-(4,5-dimethyl-2-thiazolyl)-2,5-diphenyl-2H-tetrazolium bromide (MTT) were purchased from Sigma. NP40 lysis buffer was purchased from Invitrogen (Carlsbad, CA). Polyvinylidene difluoride (PVDF) membrane was obtained from Millipore (Burlington, MA). SYBR Green I Master mix for RT-qPCR was obtained from Thermo Fisher Scientific (Waltham, MA). Qiagen RNeasy Miniprep Kit and QuantiTect Reverse Transcription Kit were purchased from Qiagen (Venlo, Netherlands).

Animals Care and Treatment. Both $A b c a 4^{-1-} R d h 8^{-/-}$with $129 \mathrm{~Sv}$ or C57BL/6 background (a generous gift from Dr. Krzysztof Palczewski, University of California Irvine, CA) (Maeda et al., 2008) and WT BALB/c mice (Jackson Laboratory, Bar Harbor, ME) at 4-6 weeks of age were used to test the protective effects of flavonoids on the bright light-induced retinal damage. $A b c a 4^{-1-} R d h 8^{-1-}$ mice were genotyped to confirm that they do not carry the retinal degeneration $8(R d 8)$ mutation, but they carry the Leu variation at amino acid 450 of retinal pigment epithelium-specific $65-\mathrm{kDa}$ protein (RPE65) (Kim et al., 2004; Gao et al., 2018). BALB/c mice were also used to determine the distribution of flavonoids in the eye upon their administration. Flavonoids were dissolved in DMSO and administered to mice by intraperitoneal injection. Both male and female mice were used in all experiments. All mice were housed in the Animal Resource Center at the School of Medicine, Case Western Reserve University and maintained in a 12-hour light/dark cycle. All animal procedures and experimental protocols were approved by the Institutional Animal Care and Use Committee at Case Western Reserve University and conformed to recommendations of both the American Veterinary Medical Association Panel on Euthanasia and the Association for Research in Vision and Ophthalmology.

Retinal Degeneration Induced with Bright Light. The $A b c a 4^{-/-} R d h 8^{-/-}$or BALB/c mice were dark-adapted 24 hours before the treatment. The flavonoid compounds at a concentration of $20 \mathrm{mg} / \mathrm{kg}$ b.wt. or DMSO vehicle were delivered to mice through intraperitoneal administration 30 minutes before exposure to bright light. Then mice pupils were dilated with $1 \%$ tropicamide, and the retinal degeneration was initiated by exposing $A b c a 4^{-/-} R d h 8^{-/-}$ mice to 10,000-lux and BALB/c mice to 12,000-lux white light delivered from a 150-W bulb (Hampton Bay; Home Depot, Atlanta, 
GA). $A b c a 4^{-1-} R d h 8^{-1-}$ mice were illuminated for 45 minutes, and $\mathrm{BALB} / \mathrm{c}$ mice were illuminated for 120 minutes (Chen et al., 2012). Retinal structures were visualized and analyzed in vivo by spectral domain-optical coherence tomography (SD-OCT) and scanning laser ophthalmology (SLO). Retinal function was examined with electroretinography (ERG). Both analyses were performed 7-10 days after bright-light exposure. Then, mice were euthanized by intraperitoneal injection of a cocktail containing ketamine $(20 \mathrm{mg} / \mathrm{ml})$ and xylazine $(1.75 \mathrm{mg} / \mathrm{ml})$ at a dose of $4 \mu \mathrm{l} / \mathrm{g}$ b.wt., and eyes were collected for preparing paraffin and cryo-sections, which were used for staining with $\mathrm{H} \& \mathrm{E}$ and immunohistochemistry, respectively. Additionally, eyes collected from different treatment groups were used for gene and protein expression analyses using RT-qPCR and immunoblotting, respectively.

SD-OCT. Ultra-high resolution SD-OCT (Bioptigen, Morrisville, $\mathrm{NC}$ ) in vivo imaging was used to evaluate the effect of flavonoids on the retinal structure in $A b c a 4^{-/-} R d h 8^{-/-}$or $\mathrm{BALB} / \mathrm{c}$ mice exposed to bright-light insult (Chen at al., 2012). Before imaging, mice pupils were dilated with $1 \%$ tropicamide and anesthetized by intraperitoneal injection of a cocktail containing ketamine $(20 \mathrm{mg} / \mathrm{ml})$ and xylazine $(1.75 \mathrm{mg} / \mathrm{ml})$ at a dose of $4 \mu \mathrm{l} / \mathrm{g}$ b.wt. The a-scan/b-scan ratio was set at 1200 lines. The OCT retinal images were obtained by scanning at 0 and $90^{\circ}$ in the b-mode. Five image frames were captured and averaged. The changes in the retinas of mice exposed to bright light and control mice were determined by measuring the outer nuclear layer (ONL) thickness $0.5-2.0 \mathrm{~mm}$ from the optic nerve head (ONH). The values of the ONL thickness were plotted using mean and S.D. Five mice were used in each experimental group, and the experiment was repeated three times.

SLO Imaging. The in vivo whole-fundus imaging of mouse retinas was obtained by using SLO (Heidelberg Engineering, Franklin, MA). Before the imaging, mice were anesthetized with ketamine, and their pupils were dilated with $1 \%$ tropicamide. SLO images were collected in the autofluorescence mode. The number of autofluorescent (AF) spots detected was counted, and the data were analyzed to determine the statistical significance. Five mice were used in each experimental group, and the experiment was repeated three times.

Retinal Histology. The effect of flavonoids on the retinal morphology in mice exposed to bright light was determined by retinal histology analysis. Eyes were collected from mice kept in the dark and treated with DMSO vehicle or flavonoid prior to illumination. Eyes were collected from euthanized mice and fixed in $10 \%$ formalin in PBS for 24 hours at room temperature (RT) on a rocking platform, and this was followed by paraffin sectioning. Sections ( $5 \mu \mathrm{m}$-thick) were stained with H\&E and imaged by a BX60 upright microscope (Olympus, Tokyo). Then the data were processed using MetaMorph software (Molecular Devices, Sunnyvale, CA).

Immunohistochemistry. To detect the expression of photoreceptor protein markers (rhodopsin and cone opsins), eyes were collected from dark-adapted mice and mice treated with DMSO vehicle or flavonoids. These eyes were fixed in $4 \%$ paraformaldehyde for 24 hours, which was followed by their incubation in $1 \%$ paraformaldehyde for 48 hours at RT, and they were then processed for cryo-sectioning. Sections $(8 \mu \mathrm{m}$-thick) were stained with a monoclonal 1D4 antirhodopsin primary antibody and Alexa Fluor 555-conjugated goat anti-mouse secondary antibody to detect rod photoreceptors. Biotinylated peanut agglutinin (PNA) and Alexa Fluor 488-conjugated streptavidin were used to detect cone photoreceptors. To detect BAX, sections were stained with polyclonal anti-Bax primary antibody and Alexa Fluor 555-conjugated goat anti-rabbit secondary antibody. Cell nuclei were detected by staining with DAPI.

Electroretinography. Retinal function was evaluated by ERG recordings performed for $A b c a 4^{-/-} R d h 8^{-/-}$or BALB/c mice 7 days after the treatment with flavonoids and illumination with bright light. Dark-adapted, not-treated mice and DMSO-treated and light-exposed mice were used as controls. Before ERG measurements, mice were anesthetized with a cocktail of $20 \mathrm{mg} / \mathrm{ml}$ ketamine and $1.75 \mathrm{mg} / \mathrm{ml}$ xylazine, and pupils were dilated with $1 \%$ tropicamide. Scotopic and photopic ERGs were recorded for both eyes of each mouse using a UTAS E-3000 universal testing and ERG system (LKC Technologies, Inc., Gaithersburg, MD). The data were processed for each condition, and ERG data were represented as mean and S.D. of both a-wave and b-wave amplitudes. Each experimental group contained five mice, and the experiment was repeated three times.

Detection of Reactive Oxygen Species In Vivo. The effect of flavonoids on the production of ROS generated in vivo after the exposure to bright light was evaluated in $A b c a 4^{-1-} R d h 8^{-1-}$ mice (Chen et al., 2012). To detect ROS, dihydroethidium (DHE), an ROSsensitive probe, was intraperitoneal injected (at a dose of $20 \mathrm{mg} / \mathrm{kg}$ b.wt.) to mice on the next day after their exposure to bright light. One hour after DHE administration, eyes were collected and fixed in a cryo-embedding medium. Cryo-sections ( $8 \mu \mathrm{m}$-thick) were used for microscopic evaluation of ROS fluorescence in the retina. The ROS fluorescence was quantified with ImageJ software (National Institutes of Health). Alternatively, to detect ROS in vivo, mice were injected with the DHE probe next day after light exposure, and 1 hour later the whole-fundus imaging in live, anesthetized mice was performed using SLO (Heidelberg Engineering) (Huber et al., 2009). Mice were anesthetized with ketamine, and their pupils were dilated with $1 \%$ tropicamide before imaging. SLO images were collected in the autofluorescence mode. The intensity of fluorescence was quantified by using ImageJ software (National Institutes of Health). We used a total 20 mice, with five mice in each experimental group.

In addition, the detection of ROS was performed in the mouse retinal lysates as described in Ezquer et al. (2016). The retinas were carefully dissected on ice, mechanically homogenized, and lysed in the cold lysis buffer (Invitrogen) containing a protease inhibitor cocktail. The equal volumes of retinal lysates were incubated with $10 \mu \mathrm{mol} / \mathrm{l}$ 2,7-dichloro-dihydro-fluorescein diacetate (Invitrogen) for 1 hour at $37^{\circ} \mathrm{C}$. Then the total fluorescence intensity in each sample was measured using a FlexStation 3 plate reader (Molecular Devices, San Jose, CA). The excitation wavelength at $485 \mathrm{~nm}$ and the emission wavelength at $520 \mathrm{~nm}$ were employed for this experiment. The fluorescence intensities were normalized per milligram of total protein. The data were expressed as a fold change obtained for each experimental group in comparison with the nontreated group. We used a total 20 mice, with five mice in each experimental group.

Detection and Quantification of Flavonoids in the Mouse Eye. High-performance liquid chromatography (HPLC) coupled to mass spectrometry (MS) analysis was used for the detection and quantification of flavonoids in the mouse eye. Flavonoids were administrated to BALB/c mice via intraperitoneal injection. Thirty minutes later, mice were euthanized to collect their eyes. Eyes from two mice from each treatment group were pooled and homogenized on ice in $1 \mathrm{ml}$ of methanol with $0.1 \%$ formic acid in the presence of 100 pmol of an internal standard (kaempferol). Then an extraction with $500 \mu \mathrm{l}$ of hexane was carried out. The polar phase was collected after centrifugation at 2,200g for 5 minutes and dried in a Savant speedvac concentrator (Thermo Fisher Scientific). The pellet was dissolved in $300 \mu \mathrm{l}$ of methanol/formic acid $0.1 \%$, and $100 \mu \mathrm{l}$ was injected into an HPLC system. Flavonoids were separated on the Hypersill Gold $50 \times$ 2.1 column (Thermo Fisher Scientific) by a linear gradient of $\mathrm{H}_{2} \mathrm{O}$ to acetonitrile $(2 \%-100 \%$ in 10 minutes and $100 \%$ acetonitrile for 5 minutes) at a flow rate of $0.35 \mathrm{ml} / \mathrm{min}$. These solvents contained $0.1 \%$ formic acid. MS-based detection and quantification of flavonoids were performed with a linear ion trap mass spectrometer (LTQ) (Thermo Fisher Scientific) equipped with an electrospray ionization interface operated in the positive ionization mode. Standards of each flavonoid were used to determine the ionization parameters and to tune the detection of the compounds. Flavonoids were detected in the selected reaction-monitoring mode using mass-to-charge ratio $(\mathrm{m} / \mathrm{z})$ for kaempferol $287.2 \rightarrow 241.1$, quercetin $303.3 \rightarrow 257.0$, and myricetin $319.2 \rightarrow 273.1$ transitions, respectively. A calibration curve was determined for each compound by calculating the linear relationship between the areas for each signal monitoring ion intensity peaks corresponding to quercetin or myricetin and kaempferol as internal 
standard versus the molar ratios of flavonoids in a range of 20-200 pmol. The experiment was performed in triplicates and repeated twice.

Cell Culture. Murine photoreceptor-derived $661 \mathrm{~W}$ cells were provided by Dr. Muayyad Al-Ubaidi, University of Houston, who developed this cell line. Cells were cultured in Dulbecco's modified Eagle's medium with 10\% FBS (Hyclone, Logan, UT) and $1 \mathrm{U} / \mathrm{ml}$ penicillin with $1 \mu \mathrm{g} / \mathrm{ml}$ streptomycin (Life Technologies) at $37^{\circ} \mathrm{C}$ under $5 \% \mathrm{CO}_{2}$ according to the received instructions.

Cytotoxicity Assay. $661 \mathrm{~W}$ cells were seeded in 96 -well plates at a density of $3 \times 10^{4}$ cells/well. The next day, the cells were exposed to different concentrations of flavonoids (quercetin or myricetin) without or with the addition of a stressor, such as $\mathrm{H}_{2} \mathrm{O}_{2}$ or all-trans-retinal. The cell viability was evaluated 24 hours later by using the MTT cell proliferation assay (Sigma). Nontreated cells were used as control. Cytotoxicity was determined by calculating the percentage of dead cells in each experimental condition. To evaluate the effect of flavonoids on the oxidative stress-induced cytotoxicity, cells plated a day before were pretreated with flavonoid at a range $(0-100 \mu \mathrm{M})$ of concentrations for 16 hours. Then $\mathrm{H}_{2} \mathrm{O}_{2}$ at $100,250,375$, or $500 \mu \mathrm{M}$ concentration was added to the cells for 24 hours. The cell viability was determined by an MTT assay. To examine the effect of flavonoids on all-trans-retinal-induced toxicity, cells that were plated a day before were treated with flavonoid or DMSO vehicle applied in a fresh medium for 16 hours followed by the addition of all-trans-retinal at different concentrations $(0-30 \mu \mathrm{M})$. The cell viability was determined by an MTT assay 24 hours later. All experimental conditions were performed in triplicate, and the experiments were repeated three times.

Quantification of Gene Expression. The effect of flavonoids on gene expression was evaluated in the mouse eyes collected from $A b c a 4^{-I-} R d h 8^{-1-}$ mice. Four groups of mice were compared: mice kept in the dark, mice treated with DMSO vehicle and exposed to light, and mice treated either with quercetin or myricetin and exposed to light. Eyes from 20 mice, with at least four mice per treatment group, were pooled together. Total RNA was isolated from the whole eye extracts by using the Qiagen RNeasy Miniprep Kit following the manufacturer's protocol. The obtained RNA samples were treated with DNase I to digest any residual chromosomal DNA. The RNA concentration was determined with a nanodrop spectrophotometer (Thermo Fisher Scientific). RNA was transcribed to cDNA by using the QuantiTect Reverse Transcription Kit (Qiagen) following the manufacturer's protocol. RT-qPCR amplification was performed using SYBR Green I Master mix (Roche Diagnostics, Mannheim, Germany) according to the manufacturer's protocol and using the StepOnePlus Real-Time PCR system (Applied Biosystems). The PCR conditions were as follows: $95^{\circ} \mathrm{C}$ for 3 minutes followed by 40 cycles of $94^{\circ} \mathrm{C}$ for 20 seconds, $55^{\circ} \mathrm{C}$ for 30 seconds, and $72^{\circ} \mathrm{C}$ for 45 seconds. Fluorescence data were acquired at the $72^{\circ} \mathrm{C}$ step. Glyceraldehyde-3-phosphate dehydrogenase (GAPDH) was used as a housekeeping gene. All data were normalized to GAPDH expression levels, and the fold changes were calculated for each gene. The amplified products were measured by amplification-curve analysis using StepOne software version 2.3. The gene expression was measured using the comparative $2-(\Delta \Delta \mathrm{Ct})$ method. PCR was performed in triplicate for each experimental condition. These experiments were repeated three times.

Alternatively, the effect of flavonoids on gene expression was evaluated in $661 \mathrm{~W}$ cells subjected to stress, such as $\mathrm{H}_{2} \mathrm{O}_{2}$ or alltrans-retinal. Twenty-four hours after treatment, cells were washed twice with PBS, collected by mechanical detachment, and resuspended in $200 \mu \mathrm{l}$ of PBS. The suspension was centrifuged at $1,000 \mathrm{~g}$ for 5 minutes. Total RNA extraction and RT-qPCR were carried out as described above. All experimental conditions were performed in triplicate, and the experiments were repeated three times.

Immunoblotting. The proteins were extracted from whole eyes collected from $\mathrm{Abca4^{-/- }} R \mathrm{dh} 8^{-/-}$mice after flavonoids treatment and light-induced damage. A total of 20 mice were used. The eyes (pooled together from four to five mice per treatment group) were mechanically homogenized in an NP40 lysis buffer (Invitrogen) containing
$1 \%$ protease inhibitor cocktail (Roche). Alternatively, protein lysates were prepared from $661 \mathrm{~W}$ cells subjected to treatments described earlier. Then the lysates were centrifuged at $12,000 \mathrm{~g}$ for 15 minutes at $4^{\circ} \mathrm{C}$. The protein concentration was measured with a BCA Protein Assay Kit (Thermo Fisher Scientific) with bovine serum albumin as a standard. The protein extract ( $60 \mu \mathrm{g} / \mathrm{lane})$ was mixed with a sample buffer, boiled for 5 minutes at $95^{\circ} \mathrm{C}$, and loaded on an SDS-PAGE gel. Alternatively, protein extract was loaded on the SDS-PAGE gel immediately after mixing with a sample buffer without boiling. The protein samples were separated with $12 \%$ SDS-PAGE gel electrophoresis and then transferred to PVDF membrane (Millipore). The PVDF membrane was probed with the following primary antibodies: anti-B-cell lymphoma 2 (BCL-2) (cat. no. A0208), anti-GAPDH (cat. no. AC002), and anti-Mef2c (cat. no. A12385) purchased from ABclonal; anti-BAX (cat. no. 182734) from Abcam; and anti-M cone opsin (cat. no. AB5405) obtained from Millipore. A horseradish peroxidase-conjugated goat anti-rabbit antibody and a horseradish peroxidase-conjugated goat anti-mouse antibody purchased from Promega (cat. no. W4011 and W4021, respectively) were used as the secondary antibody. The immunoblots were developed with the ProSignal reagents kit. GAPDH was used as the loading control. These experiments were repeated three times.

Determination of Caspase Enzymatic Activity. The activity of caspase-3 was examined with the Caspase-Glo 3/7 assay kit (Promega) according to the manufacturer's instructions. Briefly, $661 \mathrm{~W}$ cells plated on the 96-well plates were exposed to stress in the presence or absence of flavonoids. After the treatment, the media were removed, and $50 \mu \mathrm{l}$ of caspase reagent was pipetted into each well. The plate was incubated in the dark at RT for 60 minutes on the shaker before the luciferase activity was recorded using a FlexStation 3 plate reader (Molecular Devices). All experimental conditions were performed in triplicate, and the experiments were repeated three times.

Terminal Deoxynucleotidyl Transferase 2'-Deoxyuridine 5'-Triphosphate Nick-End Labeling Assay. The photoreceptor cell death was evaluated in eye cryo-sections $(8 \mu \mathrm{m})$ of $A b c a 4^{-1-} R d h 8^{-/-}$ mice that were either dark-adapted, treated with DMSO and exposed to light, or treated with flavonoid and exposed to light by terminal deoxynucleotidyl transferase 2'-deoxyuridine 5'-triphosphate nick-end labeling (TUNEL) assay and in situ cell death detection kit with fluorescein isothiocyanate (Roche Diagnostics) according to the manufacturer's protocol. This experiment was repeated 3 times.

Statistical Analyses. Values of the ONL thickness obtained from the SD-OCT images and numbers of AF spots derived from the SLO images are expressed as mean \pm S.D. Twenty mice were used per experiment, with five mice per treatment group. Each in vitro experiment was carried out in triplicates. Each experiment was repeated at least two times. The one-way ANOVA with post hoc Dunnett's test method was used for two-group comparisons. For multiple comparisons, the two-way ANOVA with Tukey's post hoc tests were used. All statistical calculations were performed using Prism GraphPad 7.02 software. Type1 error tolerance for the experiments was established at $5 \%$. The family-wise error rate for multiple comparisons was controlled with Bonferroni correction, considering the comparisons statistically different at a $P$ value of $<0.001(*)$.

\section{Results}

Flavonoids Preserve Retinal Morphology after Exposure to Intense Light in $\mathrm{Abca4}^{-/-} \mathrm{Rdhr}^{-/-}$Mice. Pathologic changes in the retinal morphology and function could be detected in $A b c a 4^{-/-} R d h 8^{-/-}$mice as soon as 7 days after exposure to light injury. Pharmacological treatments with 1) primary amines that sequester all-trans-retinal, lowering its cytotoxic concentrations in photoreceptors (Maeda et al., 2011); 2) compounds that bind to ligand-free opsin elevated after light illumination, such as 11-cis-6membered-ring-retinal (Gao et al., 2018), and the nonretinoid small molecule, YC001 (Chen et al., 2018); and 3) compounds that 
A

\begin{tabular}{|c|c|c|c|}
\hline & & Anal & lyses \\
\hline $\begin{array}{c}\text { DMSO } \\
\text { or } \\
\text { Flavonoid }\end{array}$ & $\begin{array}{l}\text { White light } \\
10,000 \text { lux }\end{array}$ & Mainteined in Dark & OCT/SLO/ERG/IHC/H\&E \\
\hline-30 min & $0 \mathrm{~min}$ & 17 & ays $/ 1$ \\
\hline
\end{tabular}
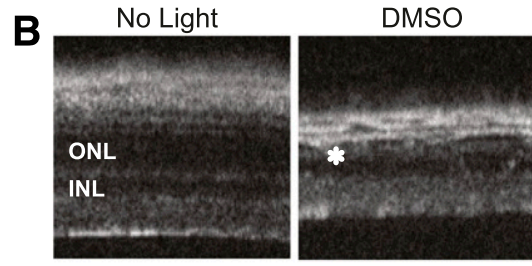

C
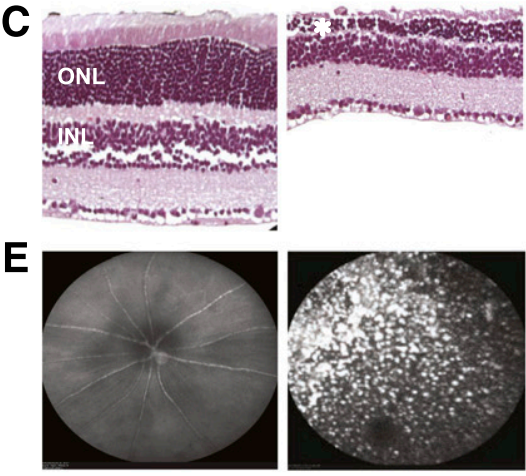

H
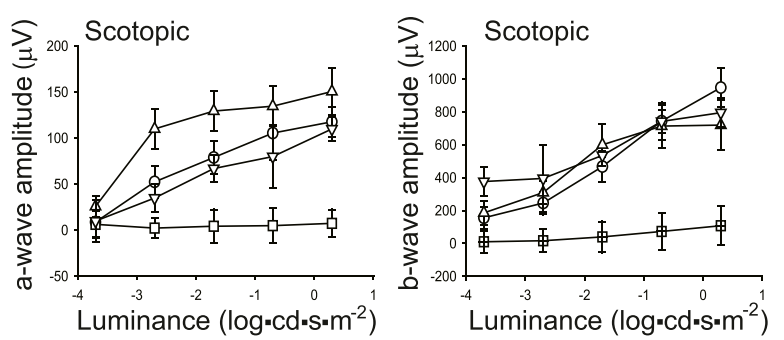
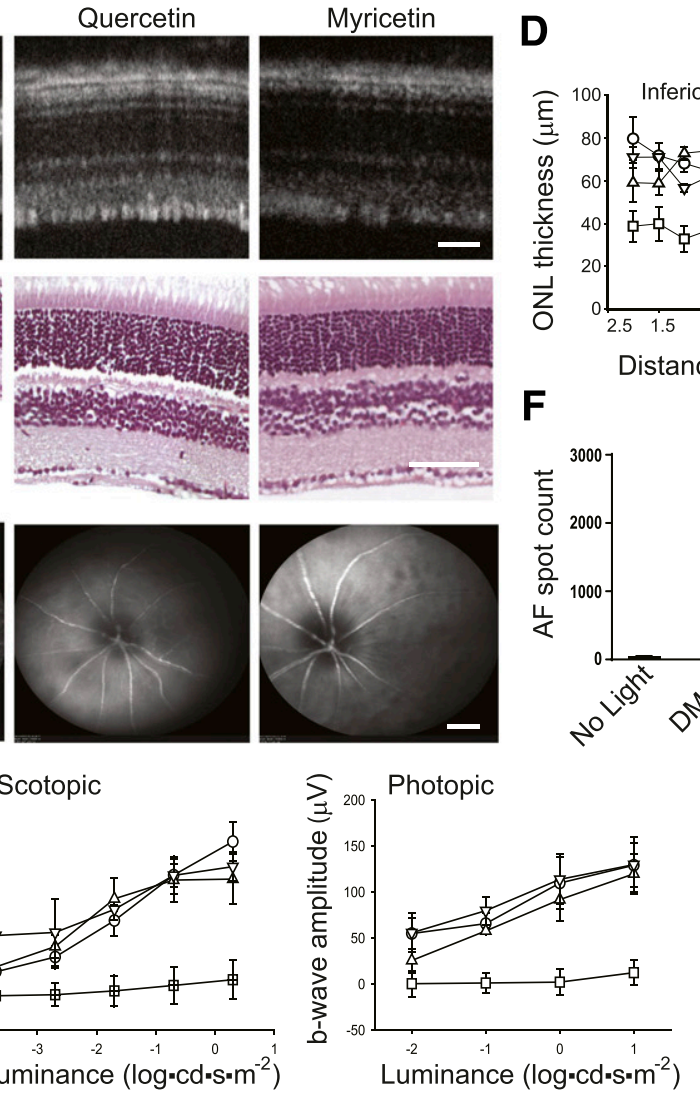

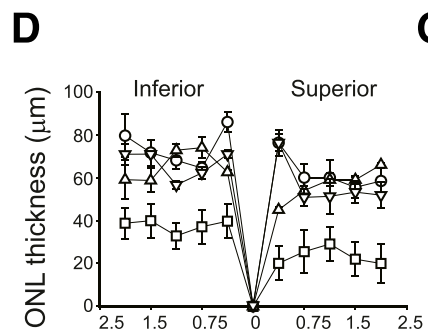

Distance from $\mathrm{ONH}(\mathrm{mm})$

\section{$\mathbf{F}$}

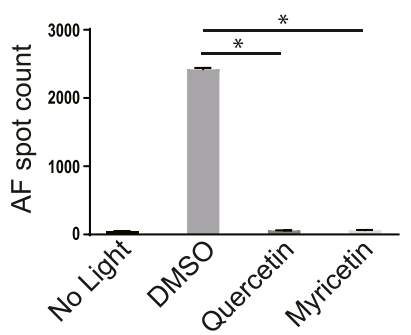

$\rightarrow$ No Light

七 DMSO

$\triangle$ Quercetin

$\rightarrow$ Myricetin
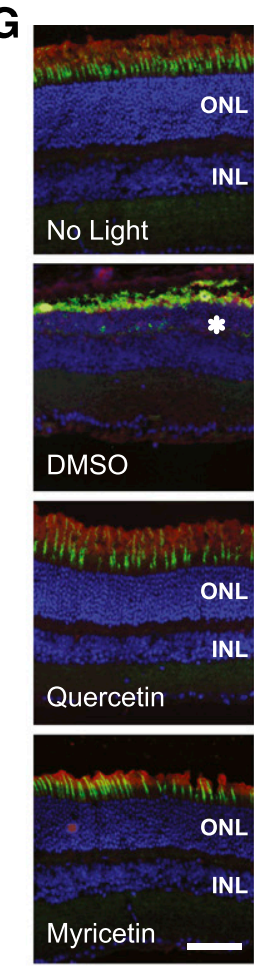

Rho/PNA/DAPI

Fig. 1. Protective effect of flavonoids against retinal degeneration in $A b c a 4^{-1-} R d h 8^{-1-}$ mice induced by bright light. (A) Experimental design of mouse treatment. Flavonoids $\left(20 \mathrm{mg} / \mathrm{kg}\right.$ b.wt.) or DMSO vehicle were administered to $4-6$-week-old $A b c a 4^{-1-} R d h 8^{-/-}$mice by intraperitoneal injection 30 minutes before exposure to 10,000-lux light for 45 minutes. After illumination, mice were kept in the dark for 7-10 days before their examination. The health of the retina was inspected by OCT and SLO in vivo imaging, H\&E staining, and immunohistochemistry. Retinal function was examined by ERG. (B) Representative OCT images of mouse eye obtained on the 7th day after the indicated treatment and exposure to bright light. INL, inner nuclear layer. Asterisk indicates a disorganized photoreceptor layer in DMSO-treated control mice. Scale bar, $50 \mu \mathrm{m}$. (C) Retinal sections prepared from eyes collected from mice either unexposed to light or exposed to bright light after the indicated treatment and stained with H\&E. Asterisk indicates disorganized photoreceptor structures in DMSO-treated control mice. Scale bar, $50 \mu \mathrm{m}$. (D) The ONL thickness was measured at inferior and superior sites at 250,500 , $750,1000,1500$, and $2000 \mu \mathrm{m}$ from the ONH. Measurements were performed in 20 mice, with five mice per treatment group. Error bars indicate S.D. Changes in the ONL thickness observed between dark-adapted group and DMSO-treated, exposed-to-light group were statistically different. Changes in the ONL thickness observed after treatment with flavonoids compared with the DMSO-treated group were statistically different. No significant difference in the ONL thickness was observed between mice kept in the dark and those treated with flavonoids. (E) Representative SLO images. AF spots were detected only in the retina of DMSO-treated and exposed-to-bright-light mice. AF spots were not detected in mice kept in the dark or mice treated with either quercetin or myricetin before illumination. Scale bar, $50 \mu \mathrm{m}$. (F) Quantification of AF spots performed in 20 mice, with five mice per treatment group. Error bars indicate S.D. Changes in the number of AF spots after treatment with flavonoids compared with DMSO-treated mice were statistically different and are indicated with asterisk $(*)$. No significant difference was observed between mice kept in the dark and those exposed to light after treatment with flavonoids. (G) Immunohistochemistry in cryo-sections prepared from eyes collected either from mice unexposed to light or exposed to bright light after the indicated treatment. Sections were stained with an anti-rhodopsin (Rho) C-terminus-specific antibody (red) showing the structural organization of rod photoreceptors, PNA staining (green) showing the health of cone photoreceptors, and DAPI staining of nuclei (blue). Asterisk shows a severely disrupted photoreceptor layer in DMSO-treated control mice. Scale bar, $50 \mu \mathrm{m}$. $(\mathrm{H})$ Retinal function examined by ERG responses. Retinal function was significantly protected in mice treated with flavonoid before exposure to bright light insult as compared with DMSO-treated mice in both scotopic a- and b-waves and in photopic b-waves. ERG measurements were carried out in 20 mice, with five mice per treatment group. Changes in the ERG responses compared between dark-adapted mice and DMSO-treated, exposed-to-light mice were statistically different. Changes in the ERG responses after treatment with flavonoids compared with DMSO-treated mice were statistically different. No statistical difference was observed between the dark-adapted group and mice treated with flavonoids. Statistical analysis was performed with the one-way ANOVA and post hoc Dunnett's tests.

reduce oxidative stress and apoptosis applied before light illumination can prevent retinal degeneration induced by acute light in these mice (Maeda et al., 2009b). Flavonoids can interact with ligand-free opsin, enhancing its stability, and they possess antioxidative and antiapoptotic properties (Ortega et al., 2019). Thus, we evaluated the effect of two dietary flavonoids, quercetin and myricetin, on light-induced retinal pathology in $A b c a 4^{-/-} R d h 8^{-/-}$mice. Flavonoids were administered to mice by i.p injection at a dose of $0.02,0.2,2.0$, or $20 \mathrm{mg} / \mathrm{kg}$ b.wt. 30 minutes before exposure to bright light. Then these mice were exposed to intense 10,000-lux light for 45 minutes, which was followed by their housing in the dark. The morphologic changes in the retinas 
TABLE 1

Dose-dependent effect of quercetin and myricetin on the retinal health in $A b c a 4^{-1-} R d h 8^{-1-}$ mice

The protective effect of flavonoids on the retinal health was evaluated after the administration of quercetin or myricetin at different doses by measuring the ONL thickness in the OCT images obtained from $A b c a 4^{-1-} R d h 8^{-1-}$ mouse eyes. After dark adaptation, mice were treated with DMSO vehicle or flavonoid at $0.02,0.2,2$, or $20 \mathrm{mg} / \mathrm{kg}$ b.wt. The flavonoid compound was administrated 30 min prior to illumination with 10,000 lux bright light. Both quercetin and myricetin were able to prevent the morphologic changes associated with light damage at concentrations higher than $2 \mathrm{mg} / \mathrm{kg}$. The percentage of protection was calculated for each treatment in comparison with "No light" group (100\% protection). The calculations were performed using the mean difference between DMSO and the specific treatment group obtained from 95\% confidence intervals (CIs). The S.E.M. difference (95\% CI) expressed as percentage was 7.54. Five mice were used for each experimental group. Statistical analysis was performed with the one-way ANOVA with Dunnett's post hoc method by using Prism GraphPad 7.02 software.

\begin{tabular}{lccc}
\hline Treatment & OCT ONL Thickness $(\mu \mathrm{m})$ & Protection of ONL Thickness $(\%)$ & Statistical Significance $(P)$ \\
\hline No light & $58 \pm 3$ & 100 & $<0.0001$ \\
DMSO & $5 \pm 2$ & 0.0 & $>0.0$ \\
Quercetin $(0.02 \mathrm{mg} / \mathrm{kg}$ b.wt.) & $5 \pm 3$ & 20.8 & 0.0312 \\
Quercetin $(0.2 \mathrm{mg} / \mathrm{kg}$ b.wt.) & $16 \pm 7$ & 35.5 & $<0.01$ \\
Quercetin $(2 \mathrm{mg} / \mathrm{kg}$ b.wt.) & $24 \pm 8$ & 98.1 & $<0.0001$ \\
Quercetin (20 mg/kg b.wt.) & $57 \pm 3$ & 0.0 & $0.99(\mathrm{NS})$ \\
Myricetin $(0.02 \mathrm{mg} / \mathrm{kg} \mathrm{b.wt.)}$ & $5 \pm 3$ & 5.7 & $0.99(\mathrm{NS})$ \\
Myiricetin $(0.2 \mathrm{mg} / \mathrm{kg}$ b.wt.) & $8 \pm 4$ & 50.9 & $<0.0001$ \\
Myricetin $(2 \mathrm{mg} / \mathrm{kg} \mathrm{b.wt.)}$ & $32 \pm 8$ & 100 & $<0.0001$ \\
Myriceitn $(20 \mathrm{mg} / \mathrm{kg}$ b.wt.) & $60 \pm 3$ & & \\
\hline
\end{tabular}

NA, not applicable; NS, not statistically significant.

induced by bright light and the effect of the applied treatment were evaluated 7 days later by in vivo imaging and histologically (Fig. 1A). The OCT imaging of the retina revealed a significant loss of the photoreceptor layer in DMSO-treated control mice (Fig. 1B). However, treatment with either quercetin or myricetin at $20 \mathrm{mg} / \mathrm{kg}$ b.wt. (but not with the smaller doses) before illumination preserved retinas against the deteriorating effect of bright light (Fig. 1B; Table 1). These in vivo observations were confirmed by histologic evaluation with H\&E staining (Fig. 1C). Indeed, the thickness of the ONL layer of the retina was significantly decreased in mice treated with DMSO vehicle and exposed to bright light, whereas pretreatment with flavonoid, either quercetin or myricetin prior to illumination, resulted in retinas closely resembling those of unexposed mice (Fig. 1D). Light-induced retinal injury is associated with the activation of microglia and macrophages that migrate to the retina to clear injured and dying photoreceptors. These immune cells could be detected in vivo in the retina as $\mathrm{AF}$ spots. In fact, an increased number of $\mathrm{AF}$ spots were detected with the SLO imaging in the fundus of DMSO-treated and illuminated-with-bright-light mice as compared with dark-adapted mice (Fig. 1, E and F). Interestingly, the administration of either quercetin or myricetin prior to light damage prevented the accumulation of $\mathrm{AF}$ spots in the retinas of these mice.

The more detailed changes in the retina induced by the exposure to bright light were determined by immunohistochemical analysis of eye cryo-sections with a specific antibody recognizing rhodopsin in rod photoreceptors and with PNA that labeled cone photoreceptors (Fig. 1G). Only residual staining of rhodopsin and cone opsin was detected in the retinas of DMSO-treated and exposed-to-light mice, whereas treatment with flavonoids resulted in the preservation of retinal morphology. The expression of rhodopsin and cone opsins detected in the flavonoid-treated mice was comparable to that detected in unexposed mice.

Treatment with Flavonoids Preserves Retinal Function in $\mathrm{Abca4}^{-/-} \mathrm{Rdh}^{-/-}$Mice Injured with Bright Light. The retinal function in $A b c a 4^{-/-} R d h 8^{-/-}$mice was determined by the ERG analysis. The mice were separated into four groups: nontreated and kept in the dark, DMSO-treated and exposed to light, and quercetin- or myricetin-treated and exposed to bright light. Both scotopic a- and b-wave and photopic b-wave responses were highly diminished in mice treated with DMSO. However, in mice treated with either quercetin or myricetin, the ERG responses were preserved and closely resembled the responses detected in nontreated, darkadapted mice (Fig. 1H). Thus, the treatment with flavonoids preserved both the retinal morphology and function in $A b c a 4^{-/-} R d h 8^{-/-}$mice exposed to bright-light stress.

Flavonoids Preserve Retinal Morphology and Function in WT Mice Exposed to Bright-Light Insult. To assess the protective effect of flavonoids against light-induced retinal damage in WT mice, we used albino BALB/c mice sensitive to excessive light that develop retinal degeneration upon exposure to intense light (LaVail et al., 1987a,b). Flavonoids were administered to these mice at the same dose (20 $\mathrm{mg} / \mathrm{kg}$ b.wt.) as the $A b c a 4^{-1-} R d h 8^{-1-}$ mice 30 minutes before their illumination with 12,000-lux light for 2 hours. Retinal morphology and function were assayed in these mice 7 days after the bright-light insult. Four groups of mice were compared: nontreated and unexposed to light, DMSO-treated, exposed to light and flavonoid (quercetin or myricetin)treated, and exposed to light. As assessed by the OCT imaging (Fig. 2A) and H\&E histologic staining (Fig. 2, B and C), mice treated with DMSO before light exposure showed a significant decrease in the thickness of the ONL layer in comparison with unexposed control mice. However, the treatment with quercetin or myricetin resulted in circumvention of this deleterious effect of intense light. In addition, as evidenced by the SLO imaging, an increase of the autofluorescence observed in DMSO-treated mice associated with the microglia and macrophage activation under bright light conditions was attenuated upon treatment with either quercetin or myricetin (Fig. 2, D and E). The responses to light stimulus evaluated by ERG revealed a beneficial effect of flavonoids on retinal function. Although ERG responses were significantly diminished in DMSO-treated and exposed-to-light mice, upon pretreatment with flavonoids, both scotopic and photopic responses were similar to those recorded in unexposed control mice (Fig. 2F). Altogether these results clearly indicate that flavonoids, such as quercetin and myricetin, possess a protective effect against bright light-induced retinopathy in both $A b c a 4^{-/-} R d h 8^{-/-}$and WT mice.

Detection of Flavonoids in the Mouse Eyes. The detection of flavonoids in biologic samples has always been 
A

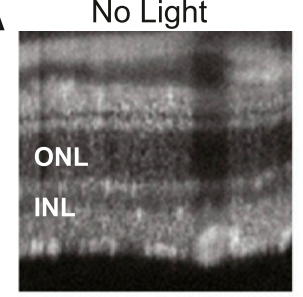

B

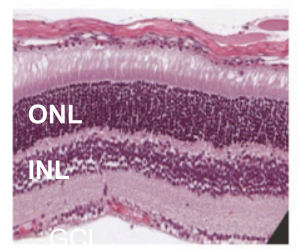

D
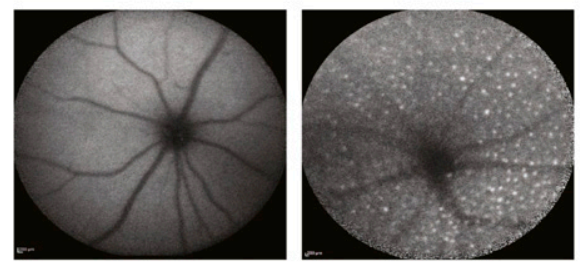

$F$

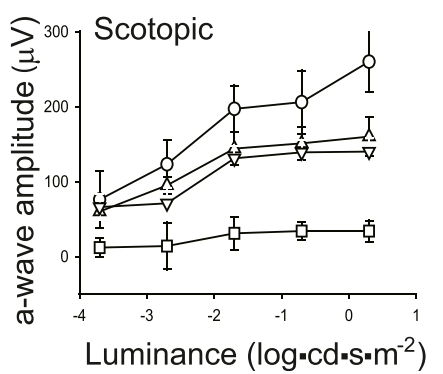

DMSO
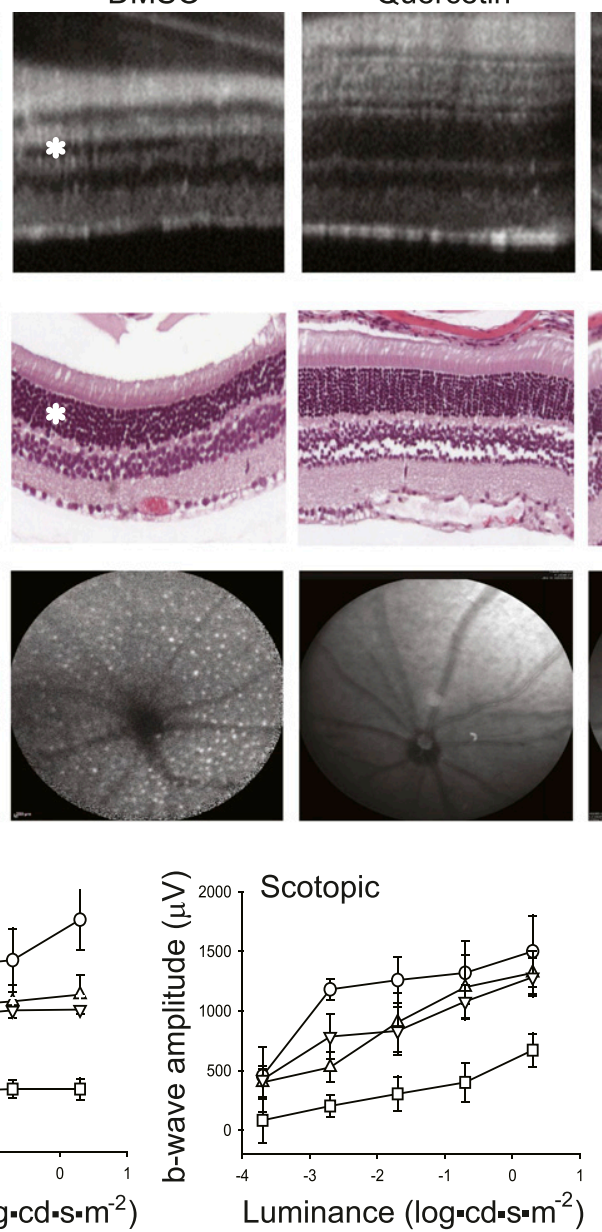

Quercetin
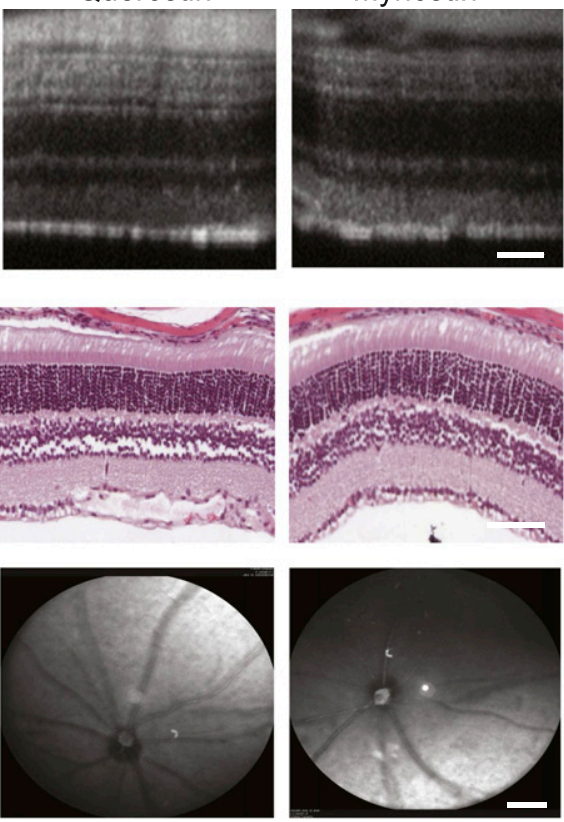

C

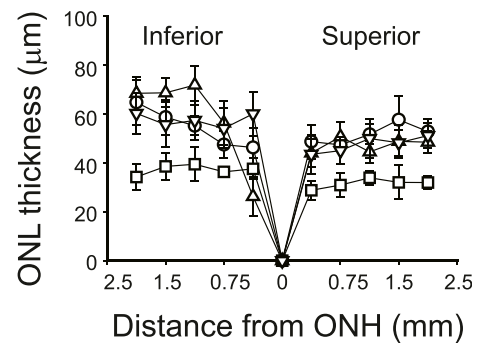

E

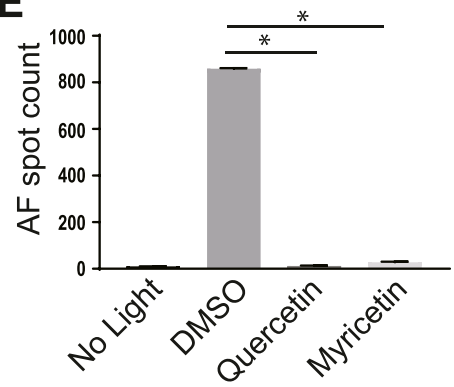

Fig. 2. The effect of flavonoids on bright light-induced retinal degeneration in WT mice. Flavonoids were intraperitoneally injected into BALB/c mice $(20 \mathrm{mg} / \mathrm{kg} \mathrm{b.wt}$.) 30 minutes before exposure to light at 12,000 lux for 2 hours. Then mice were kept in the dark for 7-10 days before examination of retinal morphology and function. (A) Representative OCT images. The ONL was protected in mice treated with either quercetin or myricetin as compared with DMSO-treated control mice. Asterisk indicates a shorter photoreceptor layer in DMSO-treated control mice. Scale bar, $50 \mu \mathrm{m}$. (B) Examination of retinal morphology after H\&E staining of paraffin sections of eyes collected from mice either kept in the dark or exposed to light after indicated treatment. Asterisk indicates a shorter photoreceptor layer in DMSO-treated control mice. Scale bar, $50 \mu \mathrm{m}$. (C) The ONL thickness measured at inferior and superior sites at $250,500,750,1000,1500$, and $2000 \mu \mathrm{m}$ from the ONH in mice kept in the dark and mice treated with DMSO vehicle or flavonoid before exposure to bright light. Measurements were performed in 20 mice, with five mice per treatment group. Error bars indicate S.D. Changes in the ONL thickness observed between dark-adapted group and DMSO-treated, exposed-to-light group were statistically different. Changes in the ONL thickness observed after treatment with flavonoids compared with the DMSO-treated group were statistically different. No significant difference in the ONL thickness was observed between mice kept in the dark and those treated with flavonoids. (D) Representative SLO images. AF spots were detected in the retina of mice treated with DMSO before exposure to bright light. Only a few AF spots were found in the retina of mice treated with flavonoid before illumination and in mice kept in the dark. Scale bar, $50 \mu \mathrm{m}$. (E) Quantification of AF spots was performed in 20 mice, with five mice per treatment group. Error bars indicate S.D. Changes in the number of AF spots compared between dark-adapted group and DMSO-treated, exposed-to-light group were statistically different. Changes in the number of AF spots after treatment with either quercetin or myricetin compared with the DMSO-treated group were statistically different and are indicated with $(*)$. No significant difference was observed between mice unexposed to light and those treated with flavonoids. (F) Retinal function examined by ERG responses. Retinal function was significantly protected in mice treated with flavonoid before exposure to bright-light insult as compared with DMSO-treated mice in both scotopic a- and b-waves and in photopic b-waves. ERG measurements were carried out in 20 mice, with five mice per treatment group. Changes in the ERG responses compared between dark-adapted mice and DMSO-treated, exposed-to-light mice were statistically different. Changes in the ERG responses after treatment with flavonoids compared with DMSO-treated mice were statistically different. No statistical difference was observed between the dark-adapted group and mice treated with flavonoids. Statistical analysis was performed with the one-way ANOVA and post hoc Dunnett's tests.

a challenge because of the chemical nature of these compounds and their low bioavailability. To learn whether quercetin and myricetin can penetrate and persist in the eye as nonmodified compounds, we performed reverse-phase HPLC-MS analysis. WT BALB/c mice were administered with a single injection of flavonoid 30 minutes before eye collection and flavonoids extraction. To quantify the amount of flavonoid that can reach the eye, we used kaempferol as an internal standard, which was added to eye homogenate before flavonoids extraction. The MS signal of kaempferol standard was found at $\mathrm{m} / \mathrm{z}=287.2[\mathrm{M}+\mathrm{H}]^{+}$, and MS/MS fragmentation at $\mathrm{m} / \mathrm{z}=241.1[\mathrm{M}+\mathrm{H}]^{+}$(Fig. 3A). In the samples extracted from mouse eyes, myricetin was detected as a nonmodified compound with an $\mathrm{m} / \mathrm{z}$ of 319.2 $[\mathrm{M}+\mathrm{H}]^{+}$, and $\mathrm{MS} / \mathrm{MS}$ fragmentation at $\mathrm{m} / \mathrm{z}=273.1[\mathrm{M}+\mathrm{H}]^{+}$. Its MS/MS fragmentation product was identical to the synthetic standard (Fig. 3B). The amount of myricetin found in the eye 30 minutes after systemic administration was $30 \pm 2.3$ pmols. However, the amount of quercetin in the examined samples was below the detection limit. Together, these results indicate that like other small 

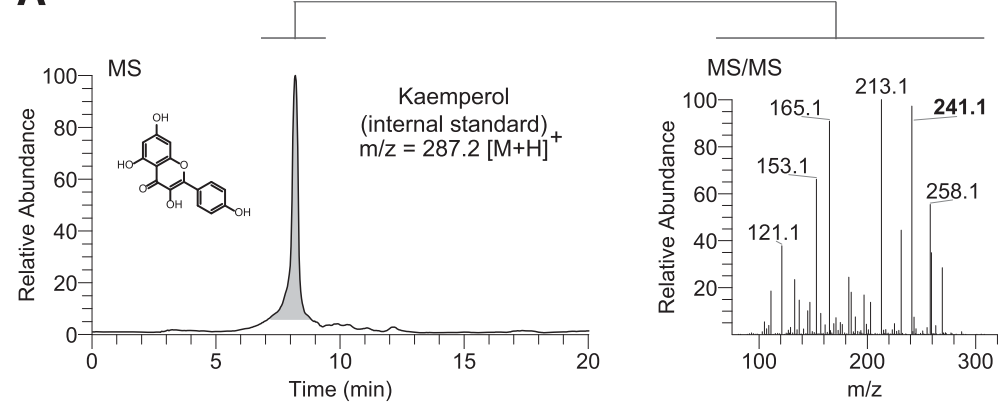

Fig. 3. Detection of flavonoids in mouse eyes. (A) MS analysis of kaemperol. Shown is the elution profile for kaemperol used as an internal standard. The chromatogram represents ion intensity for $\mathrm{m} / \mathrm{z}=287.2[\mathrm{M}+\mathrm{H}]^{+}$. Fragmentation pattern of kaemperol is shown on the right (MS/MS). (B) MS analysis of flavonoids extracted from eyes of $A b c a 4^{-1-} R d h 8^{-/-}$administered with myricetin $(20 \mathrm{mg} / \mathrm{kg}$ b.wt.). The MS spectrum of elution peak between 7 and 9 minutes (green line) indicates ion corresponding to myricetin $\mathrm{m} / \mathrm{z}=319.2[\mathrm{M}+\mathrm{H}]^{+}$. Fragmentation pattern of myricetin is shown on the right (MS/MS). The elution peak detected at $\sim 10$ minutes does not correspond with flavonoid and was also detected in the sample extracted from the eyes of nontreated control mice (NT, black line). The experiment was performed twice.
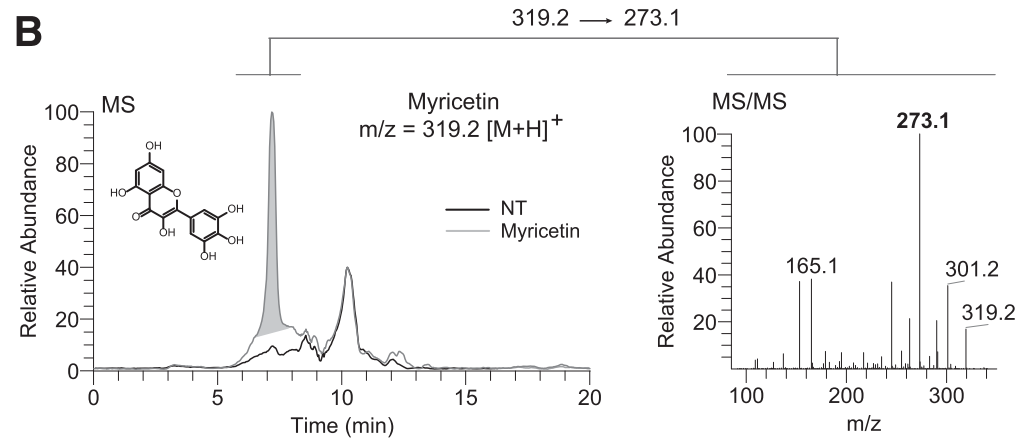

molecules, flavonoids can cross the retinal blood barrier and reach the eye.

Flavonoids Increase the Expression Level of Rhodopsin and Cone Opsins in Mice Exposed to Bright-Light Insult. Degeneration of rod and cone photoreceptors is a main pathologic factor detected in mouse retina upon exposure to intense light. The expression of rhodopsin in rod photoreceptor cells is required for the formation of outer segments, specialized structures housing this visual receptor. The levels of rhodopsin and cone opsins diminish in the bright light-injured retina because of the photoreceptors' death. Rods degenerate first, and degeneration of cones is secondary. The compounds stimulating the expression of rhodopsin and cone opsins potentially could overcome a destructive cascade leading to retina deterioration and preserve the retinal health. As reported previously, treatment with 9-cis-retinal can increase the expression of rod opsin in vitro and in vivo. In addition, recently we reported that flavonoids also enhance the expression of rod opsin and promote its membrane trafficking in cultured cells (Ortega et al., 2019). Thus, to determine whether the protective mechanism of flavonoids in mice exposed to bright light could be related to changes in the expression of opsins, we evaluated the gene and protein expression levels of rhodopsin and $\mathrm{M}$ and $\mathrm{S}$ cone opsins in $A b c a 4^{-1-} R d h 8^{-I-}$ mice treated with flavonoids prior to the exposure to bright light. Both quercetin and myricetin produced an increase in the expression levels of rhodopsin and $\mathrm{M}$ and $\mathrm{S}$ cone opsin genes as compared with DMSO-treated, illuminated mice (Fig. 4, A-C). This change in gene expression was detected as early as 1 day after illumination. On day 3 postillumination, the expression of rhodopsin was increased $\sim 2-3$-fold, M cone opsin was increased $\sim 4-5$-fold, and S cone opsin was increased 3.5-4.5-fold in flavonoid-treated mice as compared with DMSO-treated mice. The expression of these genes was even higher on day 7 and increased by $\sim 7-12$-fold for rhodopsin, 6-7fold for $\mathrm{M}$ cone opsin, and 5-fold for $\mathrm{S}$ cone opsin. The gene expression levels correlated with the protein expression levels as determined by immunoblotting with the specific antibodies

recognizing rhodopsin and $\mathrm{M}$ cone opsin (Fig. 4, E-G). The Mef2c factor is one of the key regulators of rhodopsin and cone opsins gene expression. Thus, we evaluated the effect of flavonoids on the expression of this regulatory factor. Interestingly, both quercetin and myricetin produced an increase of the gene and protein expression level of the Mef2c factor in $A b c a 4^{-1-} R d h 8^{-1-}$ mice pretreated with these flavonoids before bright-light illumination as compared with DMSOtreated and illuminated mice (Fig. 4, D, E and H). The level of Mef2c gene expression was higher by $\sim 2$-fold on day 3 and 2-3-fold on day 7 as compared with DMSO-treated mice and correlated with changes in the expression of rhodopsin and cone opsins.

Flavonoids Reduce Bright Light-Stimulated Production of Reactive Oxygen Species. Flavonoids are powerful antioxidants because of their ability to reduce and scavenge free-radical formation. Thus, the beneficial effect of flavonoids, preserving retinal health in mice exposed to bright light detrimental for photoreceptors, can be related to their capacity to decrease the cellular oxidative-stress response. In $A b c a 4^{-1-} R d h 8^{-/-}$mice, the imbalanced concentration of retinoids in the eye upon illumination with intense light triggers the excessive accumulation of ROS, leading to photoreceptor cell death (Chen et al., 2012; Gao et al., 2018). Thus, we evaluated whether quercetin and myricetin can lower the levels of ROS in the retina of $\mathrm{Abca4^{-/ }} R d h 8^{-/-}$ mice exposed to the bright-light insult. The DHE fluorescent probe injected i.p. to these mice on the next day after light illumination was used to monitor the level of ROS in the retina. In mice treated with DMSO before light exposure, an increase in ROS concentration was detected (Fig. 5, A and B; Supplemental Fig. 1). Surprisingly, in mice pretreated with quercetin before exposure to light, the level of ROS was not changed. However, in mice pretreated with myricetin, the ROS-associated fluorescence was lower than in DMSOtreated mice but not completely diminished (Fig. 5, A and B; Supplemental Fig. 1). Thus, these results suggest that the 

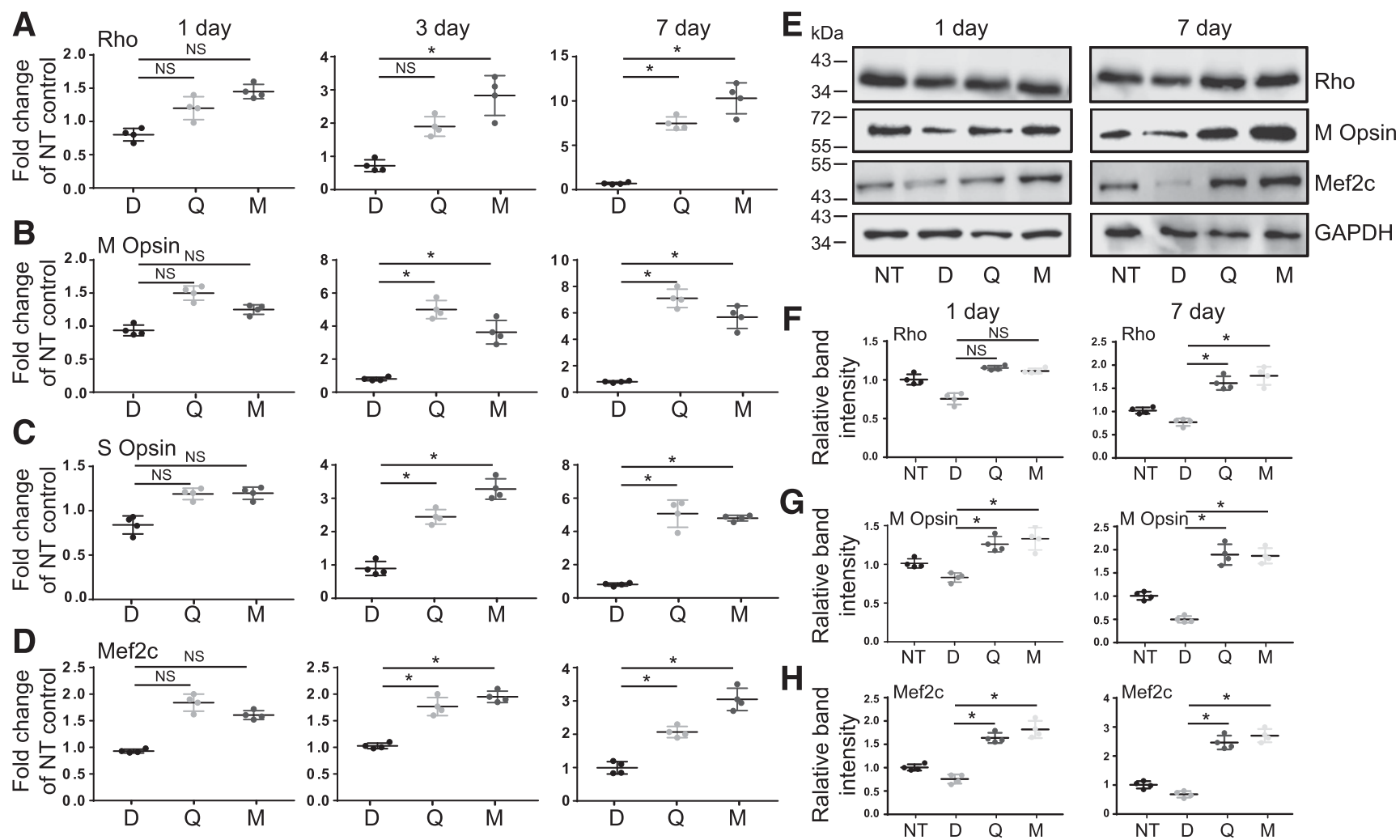

Fig. 4. The effect of flavonoids on levels of photoreceptor-specific markers in eyes of $A b c a 4^{-/-} R d h 8^{-/-}$mice exposed to bright light. Total RNA was isolated from the eyes of mice unexposed to light and mice treated with either DMSO, quercetin, or myricetin prior to illumination. Four or five mice were used for each treatment group. Eyes were collected on days 1, 3, and 7 post-treatment. RT-qPCR was performed to determine the mRNA expression levels of photoreceptor's specific genes, such as rhodopsin (Rho) (A), M cone opsin (B), S cone opsin (C), and Mef2c transcription factor regulating the expression of Rho and cone opsins (D). Relative fold change of these genes' expression was normalized to the expression of GAPDH. The mean of data from three independent experiments is shown as a fold change of NT control. The expression of these genes was slightly downregulated in DMSO-treated and illuminated mice, whereas it was significantly upregulated upon treatment with flavonoids. The statistically different changes in the expression of the specific gene compared on different days after treatment between DMSO-treated and flavonoid-treated mice are indicated with asterisk $(*)$. The nonstatistically different changes are indicated as NS. Error bars indicate S.D. Statistical analysis was performed for each gene separately, combining data for all days and using the multivariate two-way ANOVA analysis and Turkey's post hoc tests. Immunoblot analysis examining the changes in the protein expression of rhodopsin, cone opsins, and Mef2c in mouse eyes on day 1 and day 7 post-treatment. Twenty mice were used with at least four mice for each treatment group. Representative immunoblots are shown (E). Quantification of rhodopsin $(\mathrm{F}), \mathrm{M}$ cone opsin $(\mathrm{G})$, and Mef2c $(\mathrm{H})$ protein expression levels. Protein bands detected in (E) were quantified by densitometry analysis with ImageJ software. Band intensities were normalized to the intensity of GAPDH. The mean of data from three independent experiments is shown. The statistically different changes in the expression of the specific protein compared between DMSO-treated control and flavonoid-treated mice on different days are indicated with asterisk $(*)$. The nonstatistically different changes are indicated as NS. Error bars indicate S.D. Statistical analysis was performed for each gene separately, including data for all days together, using the multivariate two-way ANOVA analysis and Turkey's post hoc tests. D, treated with DMSO vehicle; M, treated with myricetin; NT, nontreated; $\mathrm{Q}$, treated with quercetin.

main mechanism of flavonoid-related protection against lightinduced retinal degeneration is not predominantly associated with their antioxidant effect in the retina. Flavonoids' protective effect against light-induced damage could rather be related to their opsin's modulatory and stabilizing properties or abilities to modulate the photoreceptor cells' own antioxidant defense mechanism. Flavonoids could function through activation of phase 2 detoxification proteins, such as, for example, heme oxygenase and glutathione $\mathrm{S}$ transferase, and Nrf2 transcription factor, which regulates the expression of these proteins (Hanneken et al., 2006; Milbury et al., 2007).

Effect of Flavonoids on the Expression of Inflammatory Markers in the Mouse Eyes Upon Bright-Light Stimulation. Exposure to bright light triggers the expression of marker genes associated with inflammation that is implicated in the pathology of photoreceptors degeneration (Rutar et al., 2015; Bian et al., 2017). The activity of flavonoids has been associated with the negative modulation of inflammatory stress markers. Because inflammatory processes are activated during the degeneration of photoreceptors, we examined whether quercetin and myricetin could downregulate the expression of several inflammatory markers in $A b c a 4^{-1-} R d h 8^{-1-}$ mice exposed to bright-light insult. The expression of chemokine 2 (CCL2), interleukin 6 (IL6), and tumor necrosis factor $\alpha(\mathrm{TNF} \alpha)$ in the mice eyes was analyzed by RT-qPCR. As shown in Fig. 5C, bright-light illumination resulted in a significant upregulation of CCL2 ( 2.5-fold), IL6 ( $\sim 3$-fold), and $\mathrm{TNF} \alpha$ ( $\sim 30$-fold) in DMSO-treated mice as compared with nontreated, unilluminated mice. However, pretreatment with quercetin or myricetin significantly reduced the expression of these markers, which closely resembled the levels observed in nontreated, dark-adapted mice. Degenerating retinal tissue features activation of microglia and macrophages, which infiltrate the subretinal space to clear dying photoreceptor cells (Kohno et al., 2013). Müller glial cells respond to acute light-induced retinal injury with enhanced expression of a glial fibrillary acidic protein (GFAP) (Chang et al., 2007). Elevated GFAP expression demonstrated 

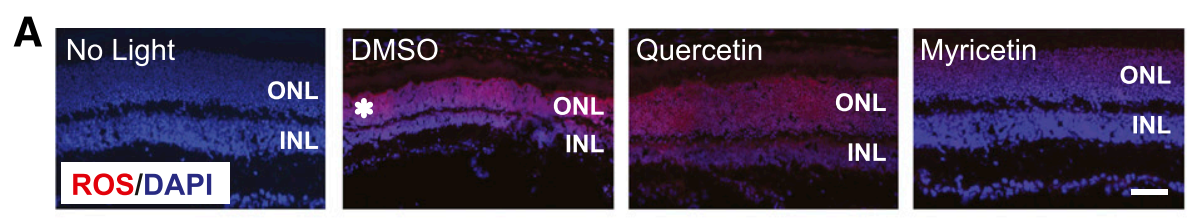

C
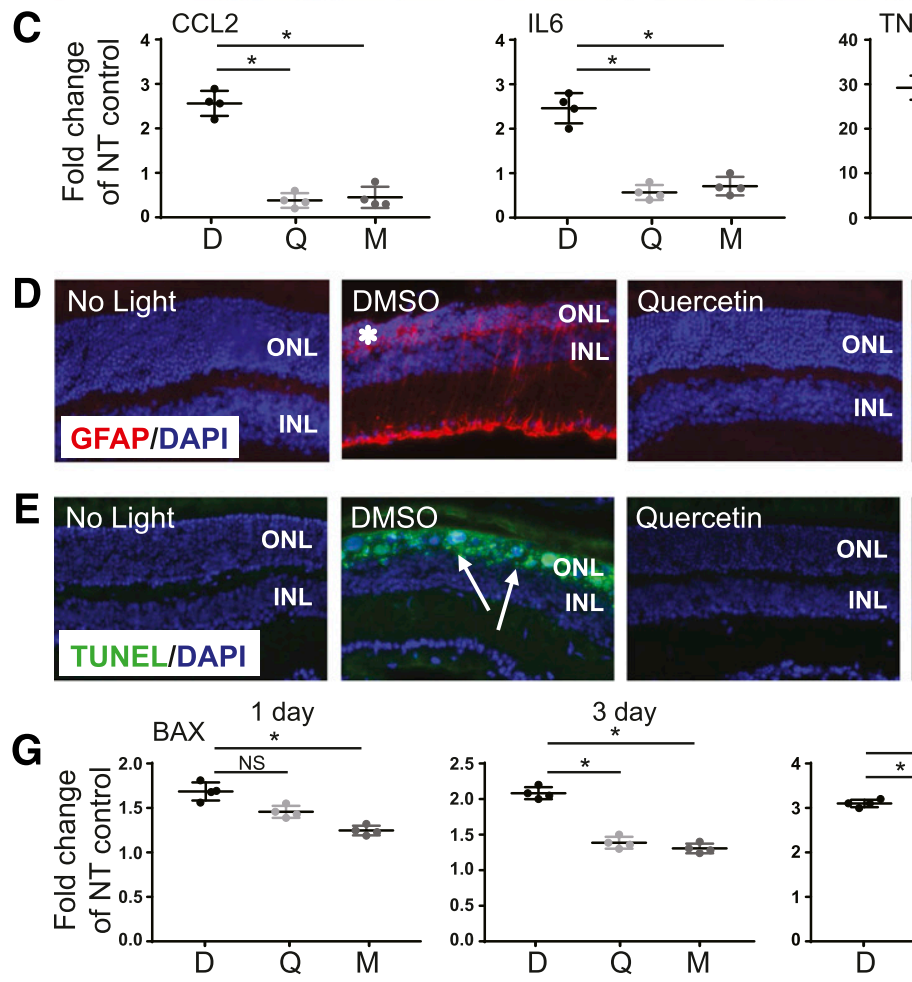

H
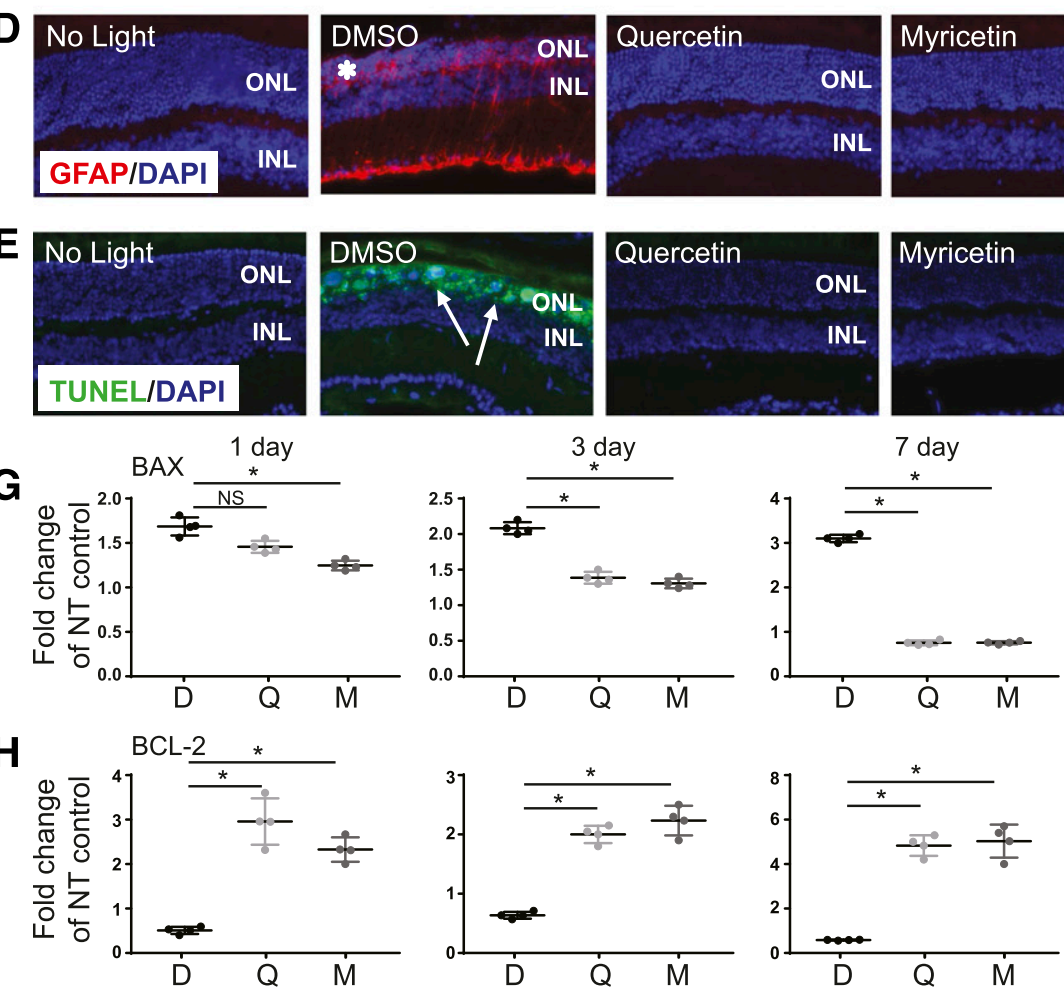

Fig. 5. The effect of flavonoids on ROS generation and levels of inflammatory and apoptotic markers in eyes of $A b c a 4^{-1-} R d h 8^{-1-}$ mice exposed to bright light. (A) Mice treated either with DMSO vehicle or flavonoid prior to exposure to 10,000-lux light or unexposed mice were injected intraperitoneally with a DHE probe 1 day after illumination. Eyes were collected 1 hour later, fixed, and processed by cryo-sectioning. The red fluorescent signal indicates ROS accumulated in the retina. Staining with DAPI (blue) was used to visualize nuclei. Scale bar, $50 \mu \mathrm{m}$. (B) Quantification of fluorescence intensity obtained from different regions of photoreceptor cell layers is presented as mean and S.D. The changes in the fluorescence intensity detected in the retina of mice treated with either quercetin or myricetin as compared with DMSO-treated and illuminated mice were not statistically different, which is indicated as NS. No changes in the fluorescence intensity were detected in the retina of mice treated with quercetin. Statistical analysis was performed with the oneway ANOVA and Dunnett's post hoc tests. (C) RT-qPCR was performed to determine the mRNA expression levels of genes implicated in inflammation. Eyes were collected from unilluminated mice and mice treated either with DMSO vehicle or flavonoid prior to illumination on day 7 post-treatment. Twenty mice were used, with at least four mice for each treatment group. Total RNA was isolated from collected mouse eyes. RT-qPCR detecting the expression levels of CCL2, IL6, and TNF $\alpha$ was performed with specific primers. Relative fold change of these genes' expression was normalized to the expression of GAPDH. The mean of data from three independent experiments is shown as the fold change of NT control. The expression of inflammatory markers was upregulated in DMSO-treated and exposed-to-light mice, whereas upon treatment with flavonoids it was significantly downregulated. The statistically different changes in the expression of the specific gene (CCL2, IL6, or TNF $\alpha$ ) compared between DMSO-treated control and flavonoid-treated mice are indicated with asterisk (*). Error bars indicate S.D. Statistical analysis was performed with the one-way ANOVA and Dunnett's post hoc tests. (D) Eye cryo-sections ( $8 \mu \mathrm{m}$-thick) were stained with an antibody against GFAP, a microglia activation marker (red). Nuclei were stained with DAPI (blue). Scale bar, $50 \mu \mathrm{m}$. GFAP positive staining was increased in DMSO-treated and light-exposed mice as compared with unilluminated mice. Treatment with flavonoids significantly reduced microglia activation induced by bright-light insult. (E) TUNEL staining performed in eye cryo-sections showed dying photoreceptors (green). Nuclei were stained with DAPI (blue). Scale bar, $50 \mu \mathrm{m}$. (F) Quantification of TUNEL-positive photoreceptor cells. The number of TUNEL-positive cells increased in DMSO-treated and illuminated mice, but it was greatly reduced in flavonoid-treated mice. The statistically different changes are indicated with asterisk (*). Error bars indicate S.D. Statistical analysis was performed with the one-way ANOVA and Dunnett's post hoc tests. (G and H) RT-qPCR performed on days 1, 3, and 7 after treatment to determine time-related changes of the mRNA expression levels of proapoptotic (BAX) and prosurvival (BCL-2) genes in mouse eyes. Twenty mice were used, with at least four mice for each treatment group. Relative fold change of these genes' expression was normalized to the expression of GAPDH. The mean of data from three independent experiments is shown as a fold change of NT control. The expression of BAX was upregulated in DMSO-treated and exposed-to-bright-light mice but was significantly downregulated upon treatment with flavonoids. The expression of BCL-2 was slightly downregulated in DMSO-treated and exposed-to-bright-light mice but was significantly upregulated upon treatment with flavonoids. The statistically significant changes in the expression of BAX and BCL-2 compared between DMSO-treated and flavonoid-treated mice on different days are indicated with $\left(^{*}\right)$. The nonstatistically different changes are indicated as NS. Error bars indicate S.D. Statistical analysis was performed for each gene separately, combining data for all days, using the multivariate two-way ANOVA 
by enhanced staining of microglial cells with an antibody against GFAP was found in the retinal cryo-sections of DMSOtreated exposed-to-bright-light $A b c a 4^{-1-} R d h 8^{-1-}$ mouse eyes as compared with unilluminated mice (Fig. 5D). The expression of GFAP was observed across the retina in the ONL, outer plexiform layer, inner nuclear layer, and inner plexiform layer. However, this aberrant expression was suppressed in mice administered with either quercetin or myricetin prior to bright-light illumination. The staining patterns were similar to those found in mice kept in the dark.

Flavonoids Modulate the BAX/BCL-2 Stoichiometry in Mouse Eyes Upon Bright-Light Injury. Prolonged exposure to bright light induces the activation of apoptotic pathways, triggering death of photoreceptors and leading to retinal deterioration. First, we checked the effect of flavonoids on photoreceptor survival using TUNEL staining (Fig. 5, E and F). TUNEL-positive dying photoreceptors were detected on eye cryo-sections of $A b c a 4^{-I-} R d h 8^{-1-}$ mice treated with DMSO and exposed to light. However, pretreatment with quercetin and myricetin before illumination protected photoreceptors from bright-light insult. In $A b c a 4^{-1-} R d h 8^{-1-}$ mice, excessive accumulation of toxic all-trans-retinal and its byproducts promote mitochondrial damage and induce activation of the apoptotic mediator BAX (Sawada et al., 2014). Elevated gene expression of BAX ( 1.7-fold) was found in mice treated with DMSO vehicle and exposed to acute light as early as 1 day after illumination, with an even greater increase on day 3 ( $\sim$-fold) and day 7 ( $\sim 3$-fold) postillumination (Fig. 5G). As reported before, compounds that inhibit the activation of BAX can rescue dying photoreceptors (Murakami et al., 2013). Moreover, mitochondria-associated apoptosis is regulated by the prosurvival protein BCL-2. An increase in BCL-2 expression results in inhibition of the apoptotic processes and promotes the survival of photoreceptors stressed by excessive light. Interestingly, the bioactivity of flavonoids is often associated with the modulation of apoptotic pathways in vitro and in vivo (Murakami et al., 2013). Thus, we examined the effect of quercetin and myricetin on expression levels of BCL-2 in the eyes of $A b c a 4^{-1-} R d h 8^{-1-}$ mice insulted with bright light. Indeed, treatment with these two flavonoids prior to bright-light exposure resulted in enhanced expression of BCL-2 (Fig. 5H). Moreover, enhanced BAX activation was detected in the eye cryo-sections of $A b c a 4^{-1-} R d h 8^{-1-}$ mice treated with DMSO vehicle and exposed to light as compared with dark-adapted mice with the specific antibody recognizing BAX. Elevated BAX expression was found in the retinal ONL and inner segments (Fig. 5I). The BAX signal was not detected in the retinas of mice treated with either quercetin or myricetin prior to light exposure. Thus, flavonoids halt deteriorative processes activated in the retina by intense light via shifting the BAX/BCL-2 equilibrium in this tissue toward the prosurvival activities of BCL-2.

Protective Effect of Flavonoids on Oxidative Stress-Induced Damage in Photoreceptor-Derived Cells In Vitro. To further understand the mechanism of flavonoids protection against light-induced retinal damage, we evaluated the effect of quercetin and myricetin in vitro in photoreceptor-derived $661 \mathrm{~W}$ cells. These cells are an immortalized cone photoreceptor cell line derived from the retinal tumor of a mouse expressing Simian Vacuolating Virus 40 (SV40) T antigen, and they have been used as a cell model for studying photo-oxidative stress and apoptosis. First, the potential toxicity of these flavonoids was examined. $661 \mathrm{~W}$ cells were exposed to a range of quercetin or myricetin (0-100 $\mu \mathrm{M}$ ) concentrations (Fig. 6, A and B, respectively). In agreement with our previous studies (Ortega et al., 2019), no toxicity of these compounds was detected after 24 hours incubation at up to $100-\mu \mathrm{M}$ concentration. Next, the oxidative stress and ROS imbalance were created in $661 \mathrm{~W}$ cells through exposure to $\mathrm{H}_{2} \mathrm{O}_{2}$ with a range of concentrations $(100-500 \mu \mathrm{M})$ (Fig. 6, C and D), which resulted in decreased cell viability from $80 \%-20 \%$ in an $\mathrm{H}_{2} \mathrm{O}_{2}$ concentration-dependent manner. However, preincubation of these cells with either quercetin or myricetin suppressed the toxic effect of $\mathrm{H}_{2} \mathrm{O}_{2}$ and enhanced the survival of these cells in a flavonoid concentration-dependent manner.

Flavonoids Prevent All-Trans-Retinal-Induced Damage in Photoreceptor-Derived Cells In Vitro. An increased local concentration of all-trans-retinal in the mouse retina upon exposure to bright light causes photoreceptors' death (Maeda et al., 2009a). Also, in vitro, incubation of the RPE-derived adult retinal pigment epithelial 19 (ARPE19) cells with all-trans-retinal at concentrations higher than $10 \mu \mathrm{M}$ induces apoptosis (Zhao et al., 2017). In this study, we examined whether flavonoids can protect photoreceptorderived $661 \mathrm{~W}$ cells from all-trans-retinal-induced toxicity. The viability of $661 \mathrm{~W}$ cells was decreased upon exposure to alltrans-retinal at 7.5-, 15-, and 30- $\mu \mathrm{M}$ concentrations (Fig. 6, E and F). However, pretreatment with flavonoid, either quercetin or myricetin, for 16 hours before adding all-trans-retinal to these cells reduced cell death in a flavonoid concentration-dependent manner. The flavonoid-mediated positive effect was effective only in the presence of lower $(7.5$ and $15 \mu \mathrm{M})$ concentrations of all-trans-retinal. However, treatment with either quercetin or myricetin failed to protect these cells exposed to the highest evaluated $(30-\mu \mathrm{M})$ concentration of all-trans-retinal.

Flavonoids Change the Expression of $M$ and S Opsin In Vitro under Oxidative Stress or All-Trans-Retinal-Induced Damage. Our recent study showed that flavonoids modulate the expression of rhodopsin in cells heterologously expressing this receptor (Ortega et al., 2019). To understand whether flavonoid compounds change the expression pattern of cone opsins in photoreceptor-derived $661 \mathrm{~W}$ cells under stress mimicking in vivo photoreceptor degeneration, these cells were pretreated with a single concentration of $100 \mu \mathrm{M}$ flavonoid for 16 hours, which was followed by their exposure to $250 \mu \mathrm{M} \mathrm{H}_{2} \mathrm{O}_{2}$ or $15 \mu \mathrm{M}$ all-trans-retinal. $\mathrm{H}_{2} \mathrm{O}_{2}$ or all-transretinal alone had no significant effect on the gene expression of $\mathrm{M}$ and $\mathrm{S}$ cone opsins (Fig. 7, A and D, respectively). However, treatment with flavonoids prior to the applied stress $\left(\mathrm{H}_{2} \mathrm{O}_{2}\right.$ or all-trans-retinal) resulted in an increased expression of both $\mathrm{M}$

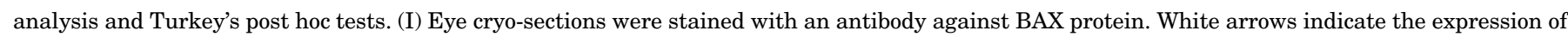

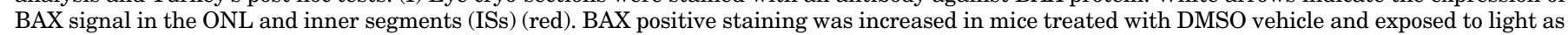

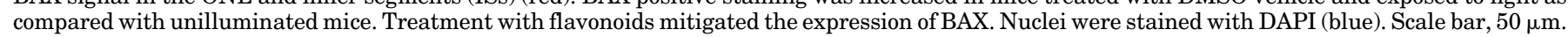
D, treated with DMSO vehicle; M, treated with myricetin; NT, nontreated; $Q$, treated with quercetin. 

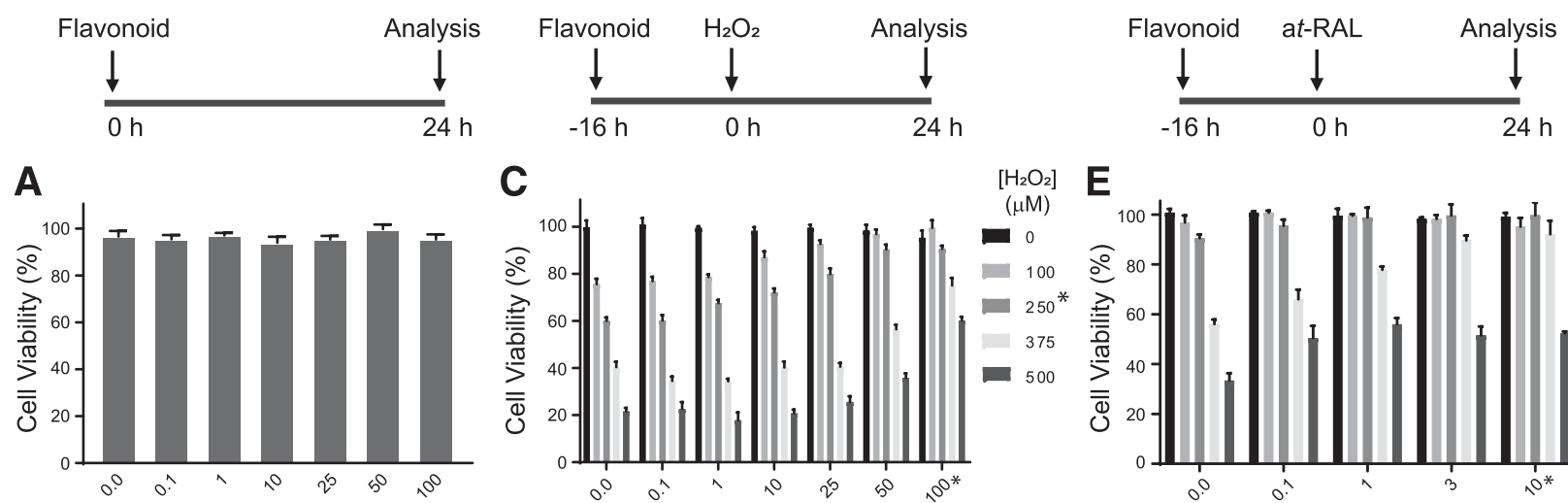

[Quercetin] $(\mu \mathrm{M})$

[Quercetin] $(\mu \mathrm{M})$
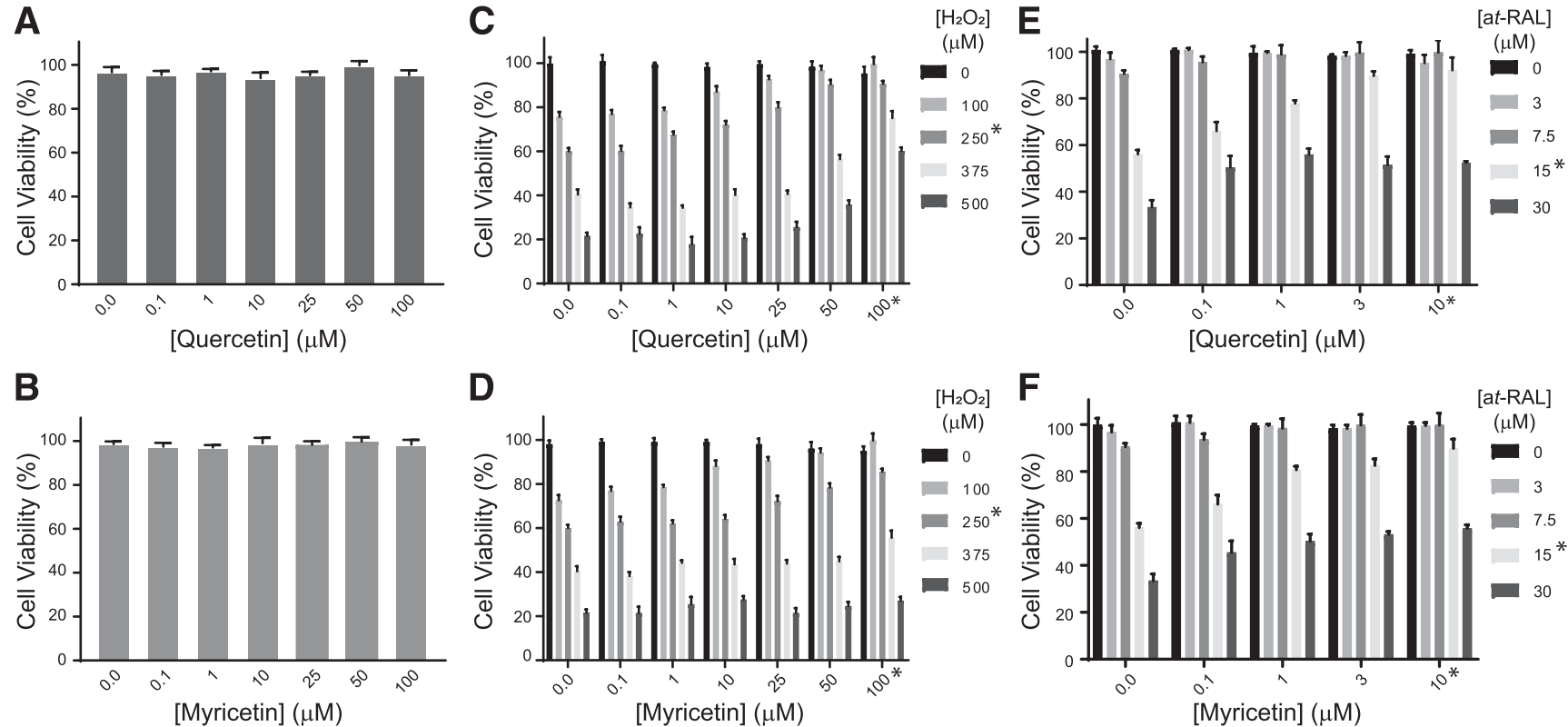

[Quercetin] $(\mu \mathrm{M})$

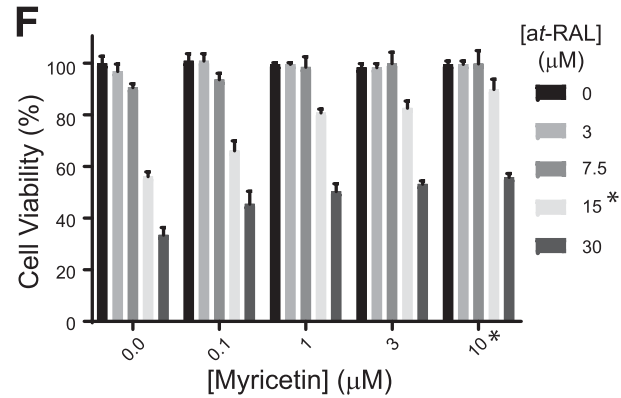

Fig. 6. The effect of flavonoids on $\mathrm{H}_{2} \mathrm{O}_{2}$ or all-trans-retinal-induced damage of $661 \mathrm{~W}$ photoreceptor-derived cells. Schemes of cell treatments with flavonoids alone, flavonoids in combination with $\mathrm{H}_{2} \mathrm{O}_{2}$, and flavonoids in combination with all-trans-retinal (at-RAL) are shown above (A, C, and E), respectively. (A and B) The effect of quercetin and myricetin, respectively, on cell viability. $661 \mathrm{~W}$ cells were treated with increasing concentrations (0-100 $\mu \mathrm{M})$ of flavonoid, and 24 hours later cell viability was evaluated with an MTT assay. No toxicity was detected. (C and D) The effect of quercetin and myricetin, respectively, on $\mathrm{H}_{2} \mathrm{O}_{2}$-induced cell damage. $661 \mathrm{~W}$ cells were treated with flavonoid at $0-100-\mu \mathrm{M}$ concentration range 16 hours before applying $\mathrm{H}_{2} \mathrm{O}_{2}$ at increasing concentrations $(100-500 \mu \mathrm{M})$. Cell viability was evaluated 24 hours later with an MTT assay. Concentration-dependent protective effect of flavonoids was noticed in cells stressed with up to $250 \mu \mathrm{M} \mathrm{H}_{2} \mathrm{O}_{2}$. (E and F) The effect of quercetin and myricetin, respectively, on all-transretinal-induced cell damage. $661 \mathrm{~W}$ cells were treated with flavonoid at $0-10-\mu \mathrm{M}$ concentration range 24 hours before applying all-trans-retinal at increasing concentrations $(0-30 \mu \mathrm{M})$. Cell viability was evaluated 24 hours after applied stress with an MTT assay. Concentration-dependent protective effect of flavonoids was noticed in cells stressed with up to $15 \mu \mathrm{M}$ all-trans-retinal. Asterisks indicate concentrations of the stressor and flavonoids used in further experiments.

and $\mathrm{S}$ cone opsin genes. Protein expression of $\mathrm{M}$ cone opsin was also elevated in cells treated with flavonoids before the applied stress as $\mathrm{H}_{2} \mathrm{O}_{2}$ (Fig. 7, B and C) or all-trans-retinal (Fig. 7, $\mathrm{E}$ and F). Interestingly, the change in the expression of cone opsins correlated with the change in the gene expression of Mef2c transcription factor, which was upregulated in cells pretreated with flavonoid before the applied stress (Fig. 7, A and D). Treatment with $\mathrm{H}_{2} \mathrm{O}_{2}$ or all-trans-retinal alone only slightly decreased Mef2c gene expression as compared with nontreated cells. However, both stressors dramatically reduced levels of Mef2c protein (Fig. 7, B, C, E, and F). Pretreatment with flavonoids before the exposure to stress prevented degradation of this protein.

Flavonoids Modulate the Survival Pathways In Vitro. Flavonoids can modulate apoptotic pathways in vitro in the retinal cell models. As shown before, apoptotic cell death induced in adult retinal pigment epithelial 19 cells either by $\mathrm{H}_{2} \mathrm{O}_{2}$ or all-trans-retinal byproduct, such as A2E, could be mitigated by treatment with flavonoids (Hanneken et al., 2006; Laabich et al., 2007; Wang et al., 2017). Activation of apoptotic processes in the cell is associated with a change in the cellular equilibrium between the proapoptotic and prosurvival markers, such as BAX and BCL-2. In this study, we evaluated the effect of quercetin and myricetin on the expression of BAX and BCL-2 in the photoreceptor-derived $661 \mathrm{~W}$ cells. Exposure of these cells to $\mathrm{H}_{2} \mathrm{O}_{2}$ or all-trans-retinal led to 1.5-2-fold increase of the gene expression of BAX. However, treatment with either quercetin or myricetin prior to $\mathrm{H}_{2} \mathrm{O}_{2}$ or all-trans-retinal exposure attenuated the expression of BAX and also led to $\sim 2.5$-fold increase in the gene expression of BCL-2 (Fig. 8, A and E, respectively). Similar effects of these flavonoids were observed on the protein levels; the expression of BAX was suppressed, whereas the expression of BCL-2 was enhanced in the cells incubated with quercetin or myricetin before $\mathrm{H}_{2} \mathrm{O}_{2}$ insult (Fig. 8, B and C) and all-trans-retinal stress (Fig. 8, F and G). Furthermore, caspase-3 is a frequently activated protease in cell apoptosis. Thus, we examined whether the activation of caspase-3 induced with $\mathrm{H}_{2} \mathrm{O}_{2}$ or all-trans-retinal in $661 \mathrm{~W}$ cells could be mitigated by flavonoids. Staurosporine, a cellpermeable alkaloid and common mediator of apoptosis, was used as a positive control. Indeed, the activity of caspase- 3 was increased in cells exposed to either $\mathrm{H}_{2} \mathrm{O}_{2}$ or all-trans-retinal as well as upon treatment with staurosporine (Fig. 8, D and H, respectively). However, pretreatment with either quercetin or myricetin prior to cell incubation with $\mathrm{H}_{2} \mathrm{O}_{2}$ or all-transretinal stressor resulted in decreased activity of this protease (Fig. 8, D and $\mathrm{H}$, respectively). Together, our results indicate that flavonoids can suppress apoptotic processes in photoreceptor-derived cells via downregulation of proapoptotic factors, such as BAX and caspase-3 and upregulation of prosurvival protein BCL-2. 
A

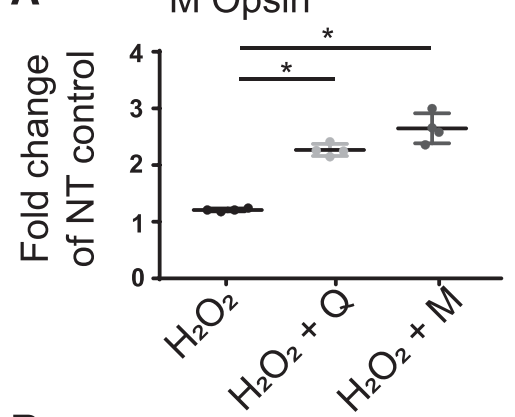

B

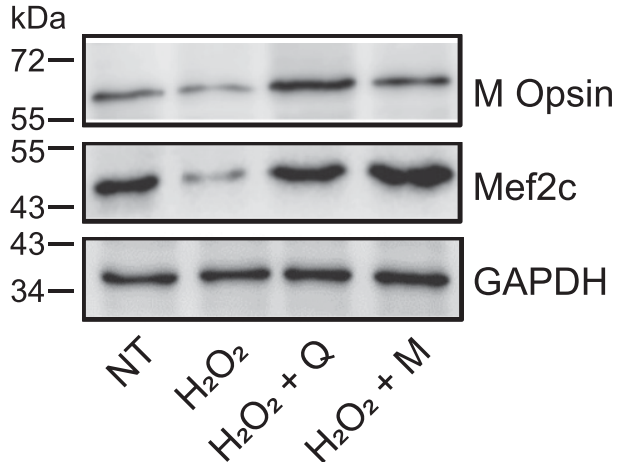

D

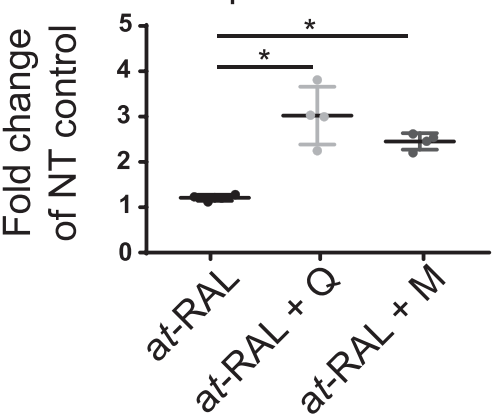

E

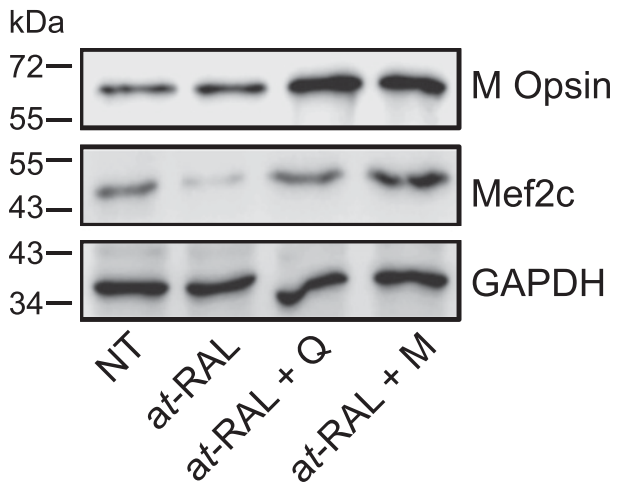

S Opsin

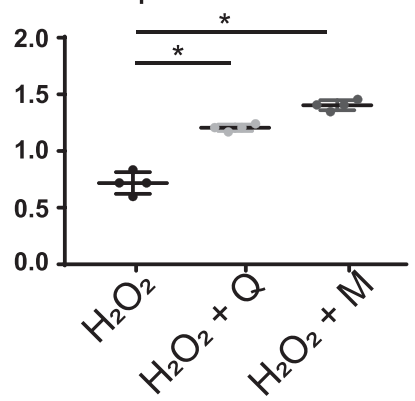

C

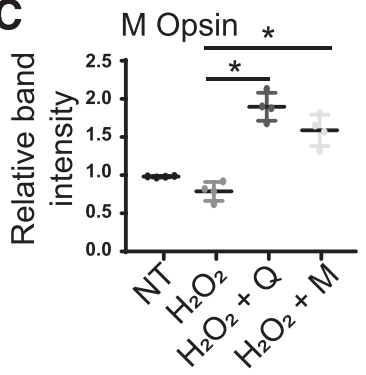

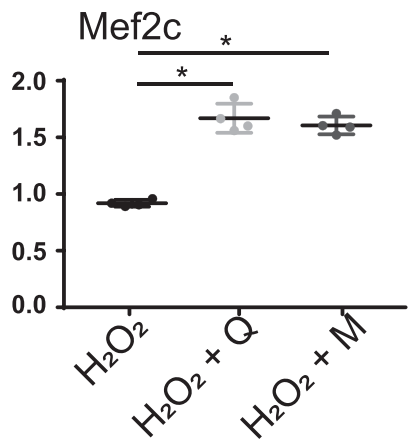

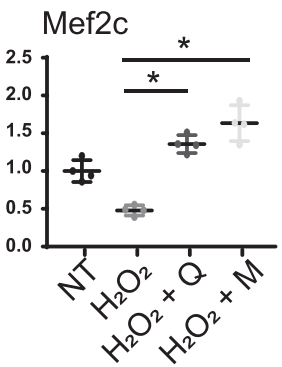

Fig. 7. The effect of flavonoids on levels of cone photoreceptor's specific markers in $661 \mathrm{~W}$ cells exposed to oxidative stress or all-trans-retinal (at-RAL). $661 \mathrm{~W}$ cells pretreated with $100 \mu \mathrm{M}$ quercetin or myricetin for 16 hours were then treated either with $\mathrm{H}_{2} \mathrm{O}_{2}(250 \mu \mathrm{M})(\mathrm{A}-\mathrm{C})$ or all-trans-retinal (15 $\left.\mu \mathrm{M}\right)$ (D-F) for 24 hours. (A and D) RT-qPCR was performed to determine the mRNA expression levels of cone-specific genes, such as M and S cone opsins, and Mef2c, their expression regulator. Relative fold change of these genes' expression was normalized to the expression of GAPDH. The mean of data from three independent experiments is shown. The expressions of cone opsins and Mef2c were upregulated in cells treated with flavonoids. The statistically significant changes in the expression of the specific gene (M and S cone opsins or Mef2c) compared with stressor $\left(\mathrm{H}_{2} \mathrm{O}_{2}\right.$ or at-RAL)-treated cells are indicated with (*). Error bars indicate S.D. Statistical analysis was performed with the one-way ANOVA and Dunnett's post hoc tests. (B and E) Immunoblot analysis examining changes in the protein expression of $\mathrm{M}$ cone opsin and Mef2c in response to stress and flavonoid treatment. (C and $\mathrm{F}$ ) Quantification of M cone opsin and Mef2c expression levels. Protein bands were quantified using densitometry analysis with ImageJ software. The mean of data from three independent experiments is shown. The statistically different changes in the expression level of the specific protein (M cone opsin or Mef2c) compared between stressor $\left(\mathrm{H}_{2} \mathrm{O}_{2}\right.$ or at-RAL)-treated cells and flavonoid-treated stressed cells are indicated with asterisk (*). Error bars indicate S.D. Statistical analysis was performed with the one-way ANOVA and Dunnett's post hoc tests. D, treated with DMSO vehicle; M, treated with myricetin; NT, nontreated; Q, treated with quercetin. 


\section{Discussion}

The visual receptor rhodopsin is a key molecule critical for retinal health (Palczewski, 2010; Jastrzebska et al., 2011). Proper morphogenesis of the retinal rod outer segments and retinal function depends on the correct expression, folding, and membrane integration of rhodopsin (Goldberg et al., 2016). Rhodopsin knockout mice do not form rod outer segments, and in rhodopsin heterozygous mice, in which the level of rhodopsin is approximately reduced by half as compared with WT mice, rod outer segments are shortened by half (Lem et al., 1999; Liang et al., 2004). This morphologic abnormality has functional implications and results in reduced responses to light signals. The energy of light captured by rhodopsin in photoreceptors is transformed into a neural signal involved in the image formation in the brain. Thus, deficiency of functional rhodopsin affects vision. The visual function of rhodopsin is tightly associated with its chromophore, 11-cis-retinal, which upon light absorption isomerizes to all-trans-retinal, triggering the formation of the rhodopsin active state necessary for signal transduction. Eventually, all-transretinal dissociates from rhodopsin to be converted back to 11-cis-retinal in the so-called retinoid (visual) cycle (Rando, 1996; Palczewski et al., 2006). Because of the high level ( $\sim 5$ $\mathrm{mM}$ ) of rhodopsin in the retina, exposure to intense light can result in free all-trans-retinal at a local concentration that is toxic to photoreceptors and RPE cells (Palczewski et al., 2006). Thus rhodopsin, although indispensable for normal retinal physiology, is also linked to various retinal pathologies. Prolonged exposure to all-trans-retinal and its byproducts is detrimental to the retina and often causes severe retinal degeneration and ultimately blindness (Travis et al., 2007; Kiser and Palczewski, 2016). In addition, excessive concentrations of unliganded opsin when regeneration of 11-cis-retinal is delayed enhance degenerative processes in the retina (Woodruff et al., 2003; Fan et al., 2005; Travis et al., 2007). The greater rate of alltrans-retinal generation upon activation of rhodopsin as compared with the rate of its reduction to all-trans-retinol typifies Stargardt diseases and AMD (Sparrow, 2016). The reactive all-trans-retinal conjugates with the membranous phosphatidylethanolamine, resulting in the formation of $\mathrm{A} 2 \mathrm{E}$ that accumulates in the RPE and causes oxidative stress in these cells. Oxidative stress and associated inflammatory responses are major pathogenic conditions contributing to retinal degeneration in Stargardt diseases and AMD (Datta et al., 2017).

In this study, we aimed to evaluate the effect of quercetin and myricetin, two common dietary flavonoids, against acute light-induced retinal degeneration. Flavonoids possess antioxidant, anti-inflammatory, and antiapoptotic properties valuable in numerous pathologies, including degenerative eye diseases (Majumdar and Srirangam, 2010; Huynh et al., 2013; Hussain et al., 2016; Bungau et al., 2019; Parmar et al., 2020). Additionally, as we recently showed, flavonoids directly interact with rhodopsin, increasing its regeneration rates, stability, folding, and membrane targeting in vitro (Herrera-Hernández et al., 2017; Ortega and Jastrzebska, 2019, Ortega et al., 2019). Thus, we hypothesized that treatment with flavonoids could prevent retinal degeneration in mouse models sensitive to brightlight insult. We used both $A b c a 4^{-1-} R d h 8^{-1-}$ mice, which lack two key enzymes of the visual cycle, a model of Stargardt disease (Maeda et al., 2008, 2014), and WT BALB/c mice susceptible to acute light retinal injury (LaVail et al., 1987a,b). These mice develop severe retinal degeneration within 1 week upon illumination with bright 10,000-12,000-lux light, which is manifested by retinal disintegration and shortening of the outer nuclear layer due to enhanced phagocytosis of dying photoreceptors. As a consequence of a decreased number of functional photoreceptors, visual responses are severely diminished in these bright light-injured mice. Interestingly, pretreatment of these mice either with quercetin or myricetin 30 minutes before the exposure to bright light preserved retinal integrity. As detected with the OCT imaging and histologic evaluation, the retinal morphology and thickness of the retinal layers closely resembled the retina of the nontreated, kept-in-the-dark mice. The morphology of both rod and cone photoreceptors was preserved in flavonoid-treated mice, indicating that flavonoids attenuated photoreceptordamaging processes induced by bright light. Notably, an oral administration of quercetin at $20 \mathrm{mg} / \mathrm{kg}$ b.wt. 48 hours, 24 hours, and 30 minutes before exposure to bright light resulted in a similar protective effect in $\mathrm{Abca4^{-1- }} R d h 8^{-/-}$ mice, emphasizing the potential prophylactic value of dietary flavonoids for retinal health. As we recently found, flavonoids stimulate expression of rhodopsin, which could have a replenishing effect preventing a reduction of its level in stressed photoreceptors, consistent with the idea that constant rhodopsin level is important for maintaining the length of rod outer segments and thus retinal health (Ortega et al., 2019). Indeed, although rhodopsin levels were reduced in retina damaged with bright light in DMSO vehicle-treated mice, the expression of rhodopsin and cone opsins was greatly enhanced upon treatment with quercetin and myricetin. The transient repression of several cone-specific genes was recently demonstrated in WT and genetically modified mice, including $\mathrm{Rpe} 65^{-/-}$and $\mathrm{Nrl}^{-1-}$ mice, suggesting that in addition to the main cause of retinal degeneration, such as the depletion of Rpe65 or $\mathrm{Nrl}$ genes, lowered expression of the visual receptors could add to the severity of this impairment (Samardzija et al., 2019). The restorative effect of flavonoids increasing the otherwise lowered level of rhodopsin was also observed in the mouse model of endotoxin-induced uveitis (Miyake et al., 2012). Moreover, the expression of Mef2c transcription factor that modulates the expression of photoreceptor-specific genes was also increased in mice treated with flavonoids, which correlated with the enhanced expression of the visual receptors. As reported earlier, knockdown of Mef2c leads to misregulation of photoreceptorspecific genes, suggesting its role in the flavonoid-stimulated enhancement of rhodopsin and cone opsins expression (Escher et al., 2011; Wolf et al., 2017). The results obtained from studies on $A b c a 4^{-l-} R d h 8^{-/-}$mouse eyes were confirmed further in photoreceptor-derived $661 \mathrm{~W}$ cells. These cells treated with quercetin and myricetin were protected from the oxidative stress or all-trans-retinal-induced cytotoxicity, likely via enhanced expression of cone opsins among other initiated prosurvival mechanisms.

The exposure to bright light potentiates ROS production in the retina (Chen et al., 2012; Miyake et al., 2012). Our results revealed elevated ROS concentrations in the retina of Abca $4^{-/-} R d h 8^{-/-}$mice illuminated with intense light. The concentration of light-induced ROS in photoreceptors was partially reduced upon treatment with myricetin, whereas quercetin had no protective effect. In general, flavonoids can limit the cellular level of ROS by directly sequestering the oxidative radicals. Quercetin can scavenge ROS in vitro at low 
A

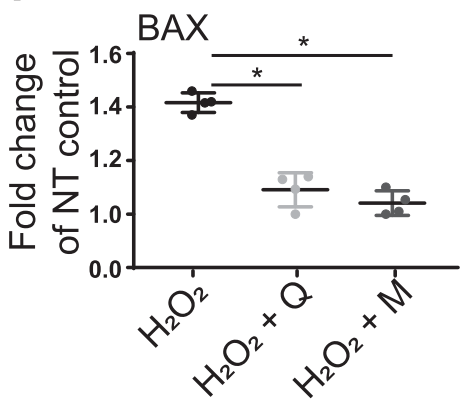

B $\mathrm{kDa}$

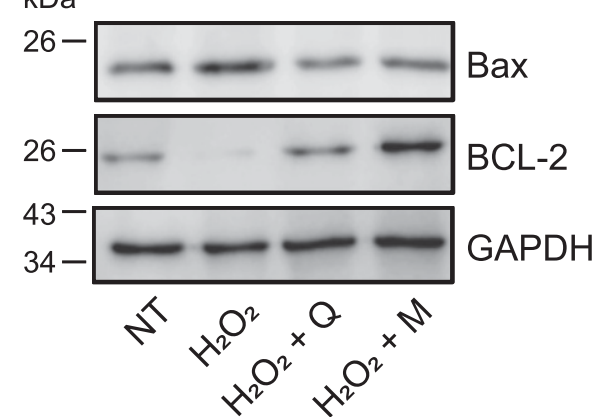

E

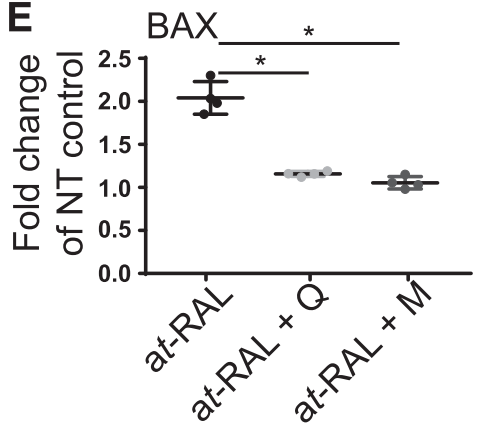

F $\mathrm{kDa}$

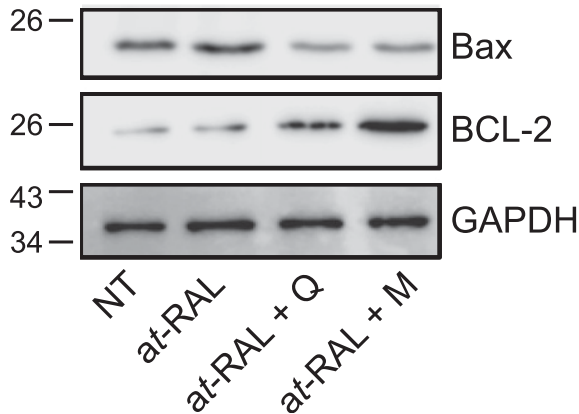

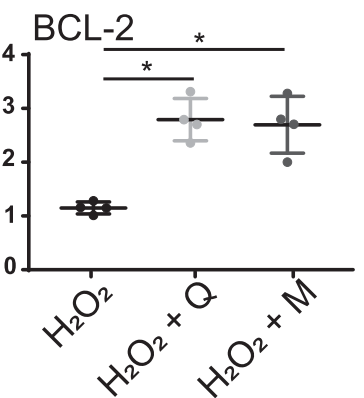

D Caspase-3 Activity

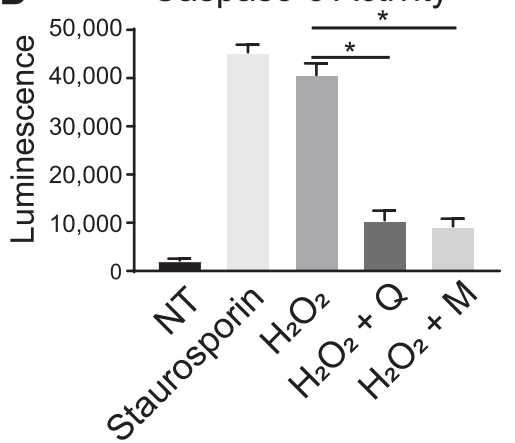

C $\quad \operatorname{Bax}$

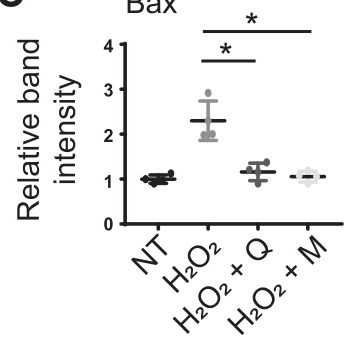

BCL-2

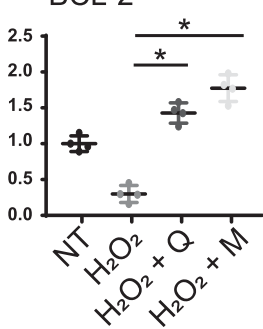

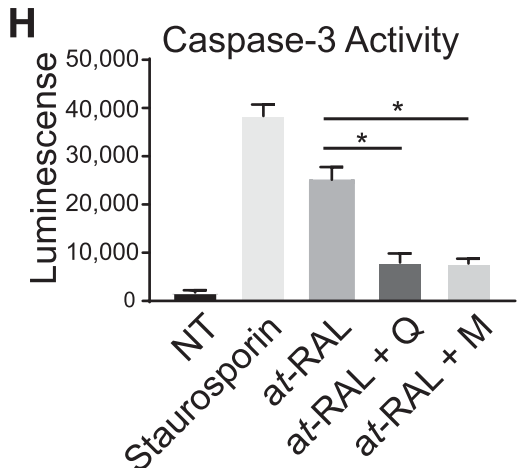

G

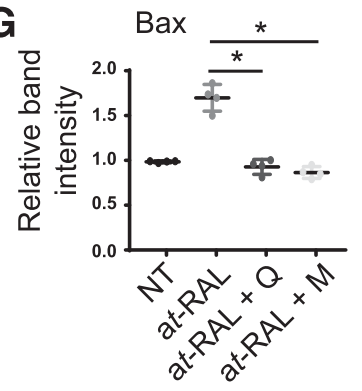

Fig. 8. The effect of flavonoids on levels of apoptotic markers in $661 \mathrm{~W}$ cells exposed to oxidative stress or all-trans-retinal (at-RAL). 661W cells pretreated with $100 \mu \mathrm{M}$ quercetin or myricetin for 16 hours were then treated either with $\mathrm{H}_{2} \mathrm{O}_{2}(250 \mu \mathrm{M})(\mathrm{A}-\mathrm{D})$ or all-trans-retinal $(15 \mu \mathrm{M})(\mathrm{E}-\mathrm{H})$ for $24 \mathrm{hours}$. (A and E) RT-qPCR was performed to determine the mRNA expression levels of genes implicated in apoptosis, such as BAX and BCL-2. Relative fold change of marker genes expression was normalized to the expression of GAPDH. The mean of data from three independent experiments is shown. The expression of BAX was downregulated, and the expression of BCL-2 was upregulated in cells treated with flavonoid. The statistically significant changes in the expression of the specific gene (BAX or BCL-2) compared with stressor $\left(\mathrm{H}_{2} \mathrm{O}_{2}\right.$ or at-RAL)-treated cells are indicated with asterisk (*). Error bars indicate S.D. Statistical significance was calculated with the one-way ANOVA and Dunnett's post hoc tests. (B and F) Immunoblot analysis examining changes in the protein expression of BAX and BCL-2 in response to stress and flavonoid treatment. Representative immunoblots are shown. (C and G) Quantification of BAX and BCL-2 protein expression. Protein bands were quantified using densitometry analysis with ImageJ software. The mean of data from three independent experiments is shown. Band intensities were normalized to the intensity of GAPDH. The statistically different changes in the expression level of the specific protein (BAX or BCL-2) between stress-treated cells and flavonoid-treated stressed cells are indicated with asterisk (*). The nonstatistically different changes are indicated as NS. Error bars indicate S.D. Statistical analysis was performed with the one-way ANOVA and Dunnett's post hoc tests. (D and H) Caspase-3 activity assay. $661 \mathrm{~W}$ cells were seeded into 96-well plates. After the indicated treatment, media was removed, and $50 \mu \mathrm{l}$ of caspase reagent was added to each well. Shown is the luciferase activity recorded after 60 minutes incubation in the dark at room temperature. The activity of caspase-3 was induced with staurosporin (positive control), $\mathrm{H}_{2} \mathrm{O}_{2}$, or all-trans-retinal, and it was significantly attenuated by treatment with flavonoid (*). Error bars indicate S.D. Statistical analysis comparing stress $\left(\mathrm{H}_{2} \mathrm{O}_{2}\right.$ or at-RAL)-treated cells with flavonoid-treated cells was performed with the one-way ANOVA and Dunnett's post hoc tests. D, treated with DMSO vehicle; M, treated with myricetin; NT, nontreated; Q, treated with quercetin. 
(5-50 $\mu \mathrm{M}$ ) micromolar concentrations (Saw et al., 2014; Barzegar, 2016; Costa et al., 2016). However, the concentration of flavonoids reaching the eye would likely be much lower in the pico to nanomolar range. Indeed, as we found in this study, by using quantitative HPLC-MS, flavonoids were detected in the mouse eyes in picomolar amounts, which likely are not enough to achieve the direct scavenging effect. Thus, the neuroprotective effect of flavonoids observed in the mouse retina was rather associated with their modulatory effects on specific cellular pathways involved in antioxidant defense mechanisms (Wang et al., 2017; Shao et al., 2019) in addition to rhodopsin-stabilizing effect (Ortega et al., 2019).

Oxidative stress activates host immune response and other defense mechanisms that under prolonged insult exacerbate photoreceptor cell death by promoting exaggerated inflammatory responses (Kohno et al., 2013; Parmar et al., 2018; Rashid et al., 2019). Flavonoids can inhibit the inflammatory reactions by suppressing the expression of proinflammatory genes and adhesion molecules implicated in the pathogenesis of retinal degeneration (Cao et al., 2010; Bian et al., 2017; Lee et al., 2017). Indeed, bright light-induced retinal inflammation in $A b c a 4^{-l-} R d h 8^{-l-}$ mice was remarkably suppressed by quercetin and myricetin, which at the molecular level correlated with the reduction of the expression of inflammatory markers.

Light-induced death of photoreceptors is also associated with activation of the proapoptotic protein BAX that is likely activated by oxidative stress and accumulated ROS (Murakami et al., 2013; Sawada et al., 2014). As we found in this study, treatment with either quercetin or myricetin before bright-light injury resulted in the negative modulation of the expression genes encoding BAX and enhanced the expression of prosurvival protein BCL-2 in the eyes of $A b c a 4^{-/-} R d h 8^{-/-}$mice. Similar effects of those two flavonoids were found in photoreceptor-derived $661 \mathrm{~W}$ cells exposed to oxidative stress or all-trans-retinal. In addition, in $661 \mathrm{~W}$ cells, treatment with flavonoids reduced the activity of caspase-3, the apoptosis executive protease. Earlier studies in cultured human RPE cells also demonstrated that quercetin can protect these cells from oxidative stress via inhibition of proinflammatory markers and inhibition of the intrinsic apoptosis pathways (Kook et al., 2008; Cao et al., 2010; Weng et al., 2017).

Together in this study, flavonoids enhanced the expression of photoreceptor-specific genes, such as rhodopsin and cone opsins, in vitro and in vivo attenuated the expression of oxidative stress and inflammation-related markers and shifted the equilibrium between the proapoptotic genes and antiapoptotic genes toward prosurvival mechanisms (Fig. 9). All these flavonoid activities collectively contribute to their protective effect halting or decelerating the retinal degeneration. Thus, cumulative experimental evidence presented here supports further evaluation of flavonoids and compounds with a flavonoid scaffold to develop pharmacological treatments against retinal degenerative disorders.

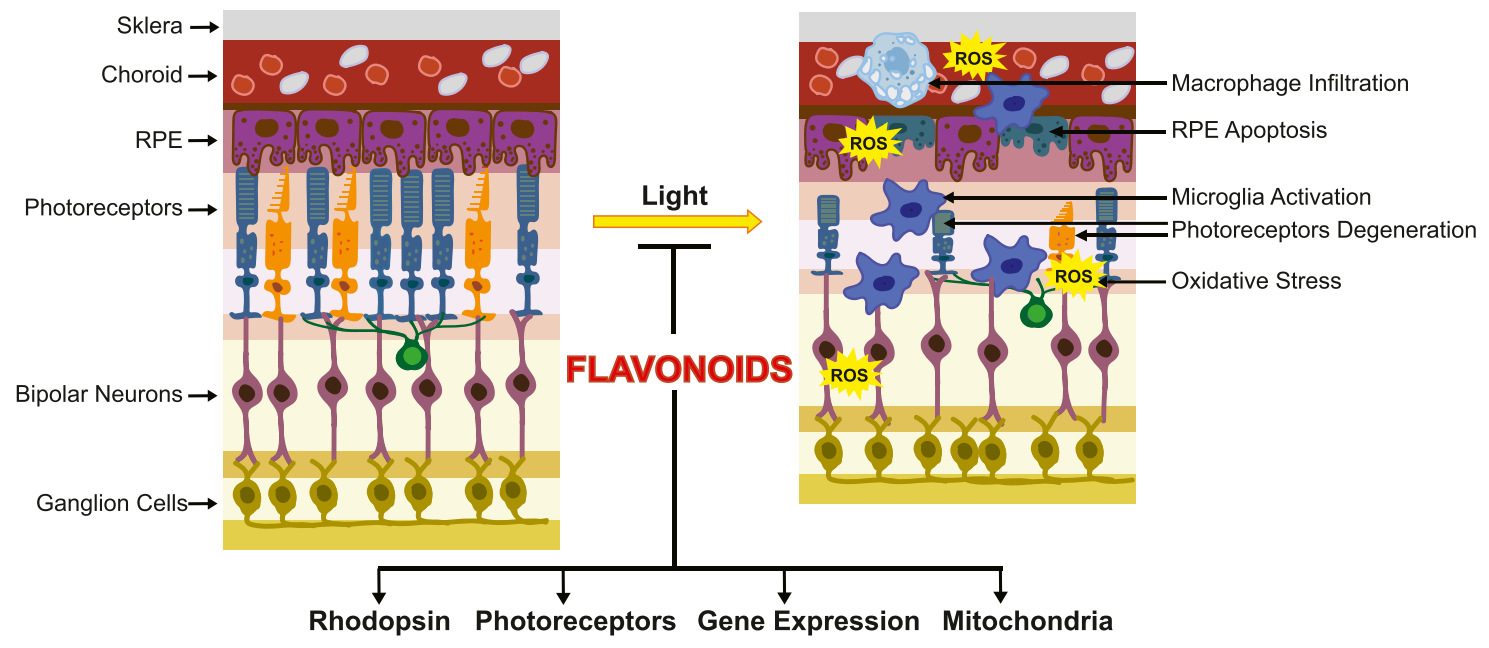

Rhodopsin Photoreceptors Gene Expression Mitochondria

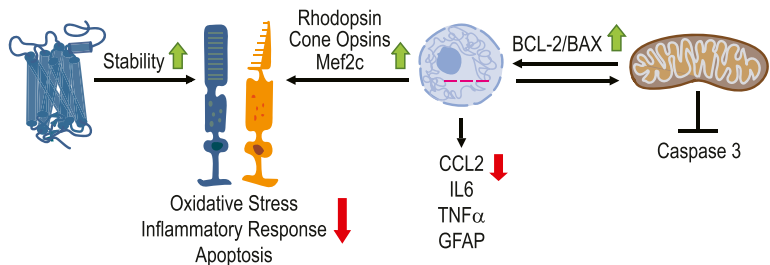

Fig. 9. The mechanism of flavonoids' protective effect on retinal health injured with bright light. The scheme of the healthy retina is shown on the left. Prolonged exposure to bright light and unfavorable genetic background lead to the degeneration of photoreceptors and RPE cells. These cells are critical to sustaining vision. Bright light triggers an overproduction of ROS leading to oxidative stress in the retina, which activates the inflammatory response in the retina orchestrated by microglia and macrophages to clear dying photoreceptors. Under persisted insult, these immune cells exacerbate the release of inflammatory mediators and exaggerate retinal damage. The scheme of the degenerated retina caused by exposure to excessive light is shown on the right. Treatment with flavonoids before the exposure to bright light prevents retinal degeneration. The mechanism of retinal health protection by flavonoids is related to a synergy of their multiple effects, such as 1) binding and stabilizing rhodopsin (Ortega et al., 2019); 2) enhancing the expression of rhodopsin and cone opsins as well as Mef2c, which regulates their expression; 3) decreasing the expression of inflammatory response mediators, such as CCL2, IL6, TNF $\alpha$, and GFAP; and 4) shifting the ratio of mitochondrial apoptotic mediators, such as BCL-2/Bax, toward the prosurvival BCL-2 and thus inhibiting the activation of the executive cell apoptosis protease, caspase-3. 


\section{Acknowledgments}

We thank Ronald E. Stenkamp for reading and editing the manuscript. The authors thank the Visual Research Science Center Core Facilities.

\section{Authorship Contributions}

Participated in research design: Ortega, Parmar, Golczak, Jastrzebska.

Conducted experiments: Ortega, Parmar, Golczak, Jastrzebska.

Performed data analysis: Ortega, Parmar, Golczak, Jastrzebska.

Wrote or contributed to the writing of the manuscript: Ortega, Golczak, Jastrzebska.

\section{References}

Barzegar A (2016) Antioxidant activity of polyphenolic myricetin in vitro cell- free and cell-based systems. Mol Biol Res Commun 5:87-95.

Bian M, Zhang Y, Du X, Xu J, Cui J, Gu J, Zhu W, Zhang T, and Chen Y (2017) Apigenin-7-diglucuronide protects retinas against bright light-induced photoreceptor degeneration through the inhibition of retinal oxidative stress and in flammation. Brain Res 1663:141-150.

Bungau S, Abdel-Daim MM, Tit DM, Ghanem E, Sato S, Maruyama-Inoue M, Yamane S, and Kadonosono K (2019) Health benefits of polyphenols and carotenoids in age-related eye diseases. Oxid Med Cell Longev 2019:9783429.

Cao X, Liu M, Tuo J, Shen D, and Chan CC (2010) The effects of quercetin in cultured human RPE cells under oxidative stress and in $\mathrm{Ccl} 2 / \mathrm{Cx} 3 \mathrm{cr} 1$ double deficient mice. Exp Eye Res 91:15-25.

Chang ML, Wu CH, Jiang-Shieh YF, Shieh JY, and Wen CY (2007) Reactive changes of retinal astrocytes and Müller glial cells in kainate-induced neuroexcitotoxicity. $J$ Anat 210:54-65.

Chen Y, Chen Y, Jastrzebska B, Golczak M, Gulati S, Tang H, Seibel W, Li X, Jin H, Han Y, et al. (2018) A novel small molecule chaperone of rod opsin and its potential therapy for retinal degeneration. Nat Commun 9:1976.

Chen Y, Okano K, Maeda T, Chauhan V, Golczak M, Maeda A, and Palczewski K (2012) Mechanism of all-trans-retinal toxicity with implications for stargardt disease and age-related macular degeneration. J Biol Chem 287:5059-5069.

Costa LG, Garrick JM, Roquè PJ, and Pellacani C (2016) Mechanisms of neuroprotection by quercetin: counteracting oxidative stress and more. Oxid Med Cell Longev 2016:2986796.

Datta S, Cano M, Ebrahimi K, Wang L, and Handa JT (2017) The impact of oxidative stress and inflammation on RPE degeneration in non-neovascular AMD. Prog Retin Eye Res 60:201-218.

Escher P, Schorderet DF, and Cottet S (2011) Altered expression of the transcription factor Mef2c during retinal degeneration in Rpe65-/- mice. Invest Ophthalmol Vis Sci 52:5933-5940.

Ezquer M, Urzua CA, Montecino S, Leal K, Conget P, and Ezquer F (2016) Intravitrea administration of multipotent mesenchymal stromal cells triggers a cytoprotective microenvironment in the retina of diabetic mice. Stem Cell Res Ther 7:42.

Fan J, Woodruff ML, Cilluffo MC, Crouch RK, and Fain GL (2005) Opsin activation of transduction in the rods of dark-reared Rpe65 knockout mice. J Physiol 568:83-95.

Fishkin NE, Sparrow JR, Allikmets R, and Nakanishi K (2005) Isolation and characterization of a retinal pigment epithelial cell fluorophore: an all-trans-retinal dimer conjugate. Proc Natl Acad Sci USA 102:7091-7096.

Gao S, Parmar T, Palczewska G, Dong Z, Golczak M, Palczewski K, and Jastrzebska B (2018) Protective effect of a locked retinal chromophore analog against lightinduced retinal degeneration. Mol Pharmacol 94:1132-1144.

Goldberg AF, Moritz OL, and Williams DS (2016) Molecular basis for photoreceptor outer segment architecture. Prog Retin Eye Res 55:52-81.

Hanneken A, Lin FF, Johnson J, and Maher P (2006) Flavonoids protect human retinal pigment epithelial cells from oxidative-stress-induced death. Invest Oph thalmol Vis Sci 47:3164-3177.

Hao H, Tummala P, Guzman E, Mali RS, Gregorski J, Swaroop A, and Mitton KP (2011) The transcription factor neural retina leucine zipper (NRL) controls photoreceptor-specific expression of myocyte enhancer factor Mef2c from an alternative promoter. J Biol Chem 286:34893-34902.

Herrera-Hernández MG, Ramon E, Lupala CS, Tena-Campos M, Pérez JJ, and Garriga P (2017) Flavonoid allosteric modulation of mutated visual rhodopsin associated with retinitis pigmentosa. Sci Rep 7:11167.

Hussain T, Tan B, Yin Y, Blachier F, Tossou MC, and Rahu N (2016) Oxidative stress and inflammation: what polyphenols can do for us? Oxid Med Cell Longev 2016 7432797.

Huynh TP, Mann SN, and Mandal NA (2013) Botanical compounds: effects on major eye diseases. Evid Based Complement Alternat Med 2013:549174.

Jastrzebska B (2013) GPCR: G protein complexes--the fundamental signaling assembly. Amino Acids 45:1303-1314.

Jastrzebska B, Debinski A, Filipek S, and Palczewski K (2011) Role of membrane integrity on $\mathrm{G}$ protein-coupled receptors: rhodopsin stability and function. Prog Lipid Res 50:267-277.

Kaarniranta K, Pawlowska E, Szczepanska J, Jablkowska A, and Blasiak J (2019) Role of mitochondrial DNA damage in ROS-mediated pathogenesis of Age-Related Macular Degeneration (AMD). Int J Mol Sci 20:2374.

Kalt W, Hanneken A, Milbury P, and Tremblay F (2010) Recent research on polyphenolics in vision and eye health. J Agric Food Chem 58:4001-4007.

Kanan Y, Jacobi AK, Sawyer K, Mannel DS, Tink JT, and Al-Ubaidi MR (2008) An invivo assay to identify compounds protective against light induced apoptosis. $A d v$ Exp Med Biol 613:61-67.
Katayama K, Gulati S, Ortega JT, Alexander NS, Sun W, Shenouda MM, Palczewski K, and Jastrzebska B (2019) Specificity of the chromophore-binding site in human cone opsins. J Biol Chem 294:6082-6093.

Kim SR, Fishkin N, Kong J, Nakanishi K, Allikmets R, and Sparrow JR (2004) Rpe65 Leu450Met variant is associated with reduced levels of the retinal pigment epithelium lipofuscin fluorophores A2E and iso-A2E. Proc Natl Acad Sci USA 101: 11668-11672.

Kim SR, Jang YP, Jockusch S, Fishkin NE, Turro NJ, and Sparrow JR (2007) The alltrans-retinal dimer series of lipofuscin pigments in retinal pigment epithelial cells in a recessive Stargardt disease model. Proc Natl Acad Sci USA 104:19273-19278.

Kiser PD, Golczak M, Maeda A, and Palczewski K (2012) Key enzymes of the retinoid (visual) cycle in vertebrate retina. Biochim Biophys Acta 1821:137-151.

Kiser PD, Golczak M, and Palczewski K (2014) Chemistry of the retinoid (visual) cycle. Chem Rev 114:194-232.

Kiser PD and Palczewski K (2016) Retinoids and retinal diseases. Annu Rev Vis Sci 2 . 197-234.

Kohno H, Chen Y, Kevany BM, Pearlman E, Miyagi M, Maeda T, Palczewski K, and Maeda A (2013) Photoreceptor proteins initiate microglial activation via Tolllike receptor 4 in retinal degeneration mediated by all-trans-retinal. J Biol Chem 288:15326-15341.

Kohno H, Maeda T, Perusek L, Pearlman E, and Maeda A (2014) CCL3 production by microglial cells modulates disease severity in murine models of retinal degeneration. J Immunol 192:3816-3827.

Kook D, Wolf AH, Yu AL, Neubauer AS, Priglinger SG, Kampik A, and Welge-Lüssen UC (2008) The protective effect of quercetin against oxidative stress in the human $\mathrm{RPE}$ in vitro. Invest Ophthalmol Vis Sci 49:1712-1720.

Laabich A, Manmoto CC, Kuksa V, Leung DW, Vissvesvaran GP, Karliga I, Kamat M, Scott IL, Fawzi A, and Kubota R (2007) Protective effects of myricetin and related flavonols against $\mathrm{A} 2 \mathrm{E}$ and light mediated-cell death in bovine retinal primary cell culture. Exp Eye Res 85:154-165.

LaVail MM, Gorrin GM, and Repaci MA (1987a) Strain differences in sensitivity to light-induced photoreceptor degeneration in albino mice. Curr Eye Res 6:825-834.

LaVail MM, Gorrin GM, Repaci MA, and Yasumura D (1987b) Light-induced retinal degeneration in albino mice and rats: strain and species differences. Prog Clin Biol Res 247:439-454.

Lee M, Yun S, Lee H, and Yang J (2017) Quercetin mitigates inflammatory responses induced by vascular endothelial growth factor in mouse retinal photoreceptor cells through suppression of nuclear factor kappa B. Int J Mol Sci 18:2497.

Lem J, Krasnoperova NV, Calvert PD, Kosaras B, Cameron DA, Nicolò M, Makino CL, and Sidman RL (1999) Morphological, physiological, and biochemical changes in rhodopsin knockout mice. Proc Natl Acad Sci USA 96:736-741.

Liang Y, Fotiadis D, Maeda T, Maeda A, Modzelewska A, Filipek S, Saperstein DA Engel A, and Palczewski K (2004) Rhodopsin signaling and organization in heterozygote rhodopsin knockout mice. J Biol Chem 279:48189-48196.

Maeda A, Golczak M, Chen Y, Okano K, Kohno H, Shiose S, Ishikawa K, Harte W, Palczewska G, Maeda T, et al. (2011) Primary amines protect against retinal degeneration in mouse models of retinopathies. Nat Chem Biol 8:170-178.

Maeda A, Maeda T, Golczak M, Chou S, Desai A, Hoppel CL, Matsuyama S, and Palczewski K (2009a) Involvement of all-trans-retinal in acute light-induced retinopathy of mice. $J$ Biol Chem 284:15173-15183.

Maeda A, Maeda T, Golczak M, and Palczewski K (2008) Retinopathy in mice induced by disrupted all-trans-retinal clearance. J Biol Chem 283:26684-26693.

Maeda A, Palczewska G, Golczak M, Kohno H, Dong Z, Maeda T, and Palczewski K (2014) Two-photon microscopy reveals early rod photoreceptor cell damage in lightexposed mutant mice. Proc Natl Acad Sci USA 111:E1428-E1437.

Maeda T, Maeda A, Matosky M, Okano K, Roos S, Tang J, and Palczewski K (2009b) Evaluation of potential therapies for a mouse model of human age-related macular degeneration caused by delayed all-trans-retinal clearance. Invest Ophthalmol Vis Sci 50:4917-4925.

Majumdar S and Srirangam R (2010) Potential of the bioflavonoids in the prevention/ treatment of ocular disorders. $J$ Pharm Pharmacol 62:951-965.

Milbury PE, Graf B, Curran-Celentano JM, and Blumberg JB (2007) Bilberry (Vaccinium myrtillus) anthocyanins modulate heme oxygenase-1 and glutathione S-transferase-pi expression in ARPE-19 cells. Invest Ophthalmol Vis Sci 48:2343-2349.

Miyake S, Takahashi N, Sasaki M, Kobayashi S, Tsubota K, and Ozawa Y (2012) Vision preservation during retinal inflammation by anthocyanin-rich bilberry extract: cellular and molecular mechanism. Lab Invest 92:102-109.

Murakami Y, Notomi S, Hisatomi T, Nakazawa T, Ishibashi T, Miller JW, and Vavvas DG (2013) Photoreceptor cell death and rescue in retinal detachment and degenerations. Prog Retin Eye Res 37:114-140.

Ortega JT and Jastrzebska B (2019) The retinoid and non-retinoid ligands of the rod visual G protein-coupled receptor. Int J Mol Sci 20:6218.

Ortega JT, Parmar T, and Jastrzebska B (2019) Flavonoids enhance rod opsin stability, folding, and self-association by directly binding to ligand-free opsin and modulating its conformation. J Biol Chem 294:8101-8122.

Palczewski K (2010) Retinoids for treatment of retinal diseases. Trends Pharmacol Sci 31:284-295.

Palczewski K, Hofmann KP, and Baehr W (2006) Rhodopsin--advances and perspectives. Vision Res 46:4425-4426.

Park PS, Lodowski DT, and Palczewski K (2008) Activation of G protein-coupled receptors: beyond two-state models and tertiary conformational changes. Annu Rev Pharmacol Toxicol 48:107-141.

Parmar T, Ortega JT, and Jastrzebska B (2020) Retinoid analogs and polyphenols as potential therapeutics for age-related macular degeneration. Exp Biol Med (Maywood) 245:1615-1625.

Parmar T, Parmar VM, Perusek L, Georges A, Takahashi M, Crabb JW, and Maeda A (2018) Lipocalin 2 plays an important role in regulating inflammation in retina degeneration. J Immunol 200:3128-3141.

Patel R, Varghese JF, Singh RP, and Yadav UCS (2019) Induction of endothelial dysfunction by oxidized low-density lipoproteins via downregulation of Erk-5/ Mef2c/KLF2 signaling: amelioration by fisetin. Biochimie 163:152-162. 
Rando RR (1996) Polyenes and vision. Chem Biol 3:255-262.

Rashid K, Akhtar-Schaefer I, and Langmann T (2019) Microglia in retinal degeneration. Front Immunol 10:1975.

Rashid K, Wolf A, and Langmann T (2018) Microglia activation and immunomodulatory therapies for retinal degenerations. Front Cell Neurosci 12:176.

Rutar M, Natoli R, Chia RX, Valter K, and Provis JM (2015) Chemokine-mediated inflammation in the degenerating retina is coordinated by Müller cells, activated microglia, and retinal pigment epithelium. J Neuroinflammation 12:8,

Samardzija M, Todorova V, Gougoulakis L, Barben M, Nötzli S, Klee K, Storti F, Gubler A, Imsand C, and Grimm C (2019) Light stress affects cones and horizontal cells via rhodopsin-mediated mechanisms. Exp Eye Res 186:107719.

Saw CL, Guo Y, Yang AY, Paredes-Gonzalez X, Ramirez C, Pung D, and Kong AN (2014) The berry constituents quercetin, kaempferol, and pterostilbene synergistically attenuate reactive oxygen species: involvement of the Nrf2-ARE signaling pathway. Food Chem Toxicol 72:303-311.

Sawada O, Perusek L, Kohno H, Howell S.J, Maeda A, Matsuyama S, and Maeda T (2014) All-trans-retinal induces Bax activation via DNA damage to mediate retinal cell apoptosis. Exp Eye Res 123:27-36.

Shao Y, Yu H, Yang Y, Li M, Hang L, and Xu X (2019) A solid dispersion of quercetin shows enhanced Nrf2 activation and protective effects against oxidative injury in a mouse model of dry age-related macular degeneration. Oxid Med Cell Longev 2019:1479571.

Sharma AK and Rohrer B (2007) Sustained elevation of intracellular cGMP causes oxidative stress triggering calpain-mediated apoptosis in photoreceptor degeneration. Curr Eye Res 32:259-269.

Sparrow JR (2016) Vitamin A-aldehyde adducts: AMD risk and targeted therapeutics. Proc Natl Acad Sci USA 113:4564-4569.
Sparrow JR, Wu Y, Kim CY, and Zhou J (2010) Phospholipid meets all-trans-retinal: the making of RPE bisretinoids. J Lipid Res 51:247-261.

Travis GH, Golczak M, Moise AR, and Palczewski K (2007) Diseases caused by defects in the visual cycle: retinoids as potential therapeutic agents. Annu Rev Pharmacol Toxicol 47:469-512.

Wang Y, Kim HJ, and Sparrow JR (2017) Quercetin and cyanidin-3-glucoside protect against photooxidation and photodegradation of $\mathrm{A} 2 \mathrm{E}$ in retinal pigment epithelial cells. Exp Eye Res 160:45-55.

Weng S, Mao L, Gong Y, Sun T, and Gu Q (2017) Role of quercetin in protecting ARPE19 cells against $\mathrm{H} 2 \mathrm{O} 2$-induced injury via nuclear factor erythroid 2 like 2 pathway activation and endoplasmic reticulum stress inhibition. Mol Med Rep 16:3461-3468.

Wolf A, Aslanidis A, and Langmann T (2017) Retinal expression and localization of Mef2c support its important role in photoreceptor gene expression. Biochem Biophys Res Commun 483:346-351.

Woodruff ML, Wang Z, Chung HY, Redmond TM, Fain GL, and Lem J (2003) Spontaneous activity of opsin apoprotein is a cause of Leber congenital amaurosis. Nat Genet 35:158-164

Zhao J, Liao Y, Chen J, Dong X, Gao Z, Zhang H, Wu X, Liu Z, and Wu Y (2017) Aberrant buildup of all-trans-retinal dimer, a nonpyridinium bisretinoid lipofuscin fluorophore, contributes to the degeneration of the retinal pigment epithelium. Invest Ophthalmol Vis Sci 58:1063-1075.

Address correspondence to: Dr. Beata Jastrzebska, Department of Pharmacology, School of Medicine, Case Western Reserve University, 10900 Euclid Ave., Cleveland, OH 44106-4965. E-mail: bxj27@case.edu 